\title{
AN EVOLUTIONARY PARADIGM FOR DUSTY ACTIVE GALAXIES AT LOW REDSHIFT
}

\author{
D. Farrah ${ }^{1,2}$, B. Connolly ${ }^{3}$, N. Connolly ${ }^{4}$, H. W. W. Spoon $^{2}$, S. Oliver ${ }^{1}$, H. B. Prosper ${ }^{5}$, L. Armus ${ }^{6}$, J. R. Houck ${ }^{2}$, \\ A. R. LIDDLE ${ }^{1}$, AND V. DESAI ${ }^{6}$ \\ ${ }^{1}$ Astronomy Centre, University of Sussex, Brighton, UK; D.Farrah@sussex.ac.uk \\ ${ }^{2}$ Astronomy Department, Cornell University, Ithaca, NY 14853, USA \\ ${ }^{3}$ Department of Physics and Astronomy, University of Pennsylvania, Philadelphia, PA 19104-6396, USA \\ ${ }_{4}^{4}$ Physics Department, Hamilton College, Clinton, NY 13323, USA \\ ${ }^{5}$ Department of Physics, Florida State University, Tallahassee, FL 32306, USA \\ ${ }^{6}$ Spitzer Science Center, California Institute of Technology, 1200 East California Boulevard, Pasadena, CA 91125, USA \\ Received 2008 December 1; accepted 2009 May 13; published 2009 July 2
}

\begin{abstract}
We apply methods from Bayesian inferencing and graph theory to a data set of 102 mid-infrared spectra, and archival data from the optical to the millimeter, to construct an evolutionary paradigm for $z<0.4$ infraredluminous galaxies. We propose that the ultraluminous infrared galaxies (ULIRG) lifecycle consists of three phases. The first phase lasts from the initial encounter until approximately coalescence. It is characterized by homogeneous mid-IR spectral shapes, and IR emission mainly from star formation, with a contribution from an active galactic nucleus (AGN) in some cases. At the end of this phase, a ULIRG enters one of two evolutionary paths depending on the dynamics of the merger, the available quantities of gas, and the masses of the black holes in the progenitors. On one branch, the contributions from the starburst and the AGN to the total IR luminosity decline and increase, respectively. The IR spectral shapes are heterogeneous, likely due to feedback from AGN-driven winds. Some objects go through a brief QSO phase at the end. On the other branch, the decline of the starburst relative to the AGN is less pronounced, and few or no objects go through a QSO phase. We show that the $11.2 \mu \mathrm{m}$ polycyclic aromatic hydrocarbon feature is a remarkably good diagnostic of evolutionary phase, and identify six ULIRGs that may be archetypes of key stages in this lifecycle.
\end{abstract}

Key words: galaxies: active - galaxies: evolution - galaxies: interactions -infrared: galaxies - methods: data analysis - methods: statistical

Online-only material: color figures

\section{INTRODUCTION}

Ultraluminous infrared galaxies (ULIRGS, objects with restframe $1-1000 \mu$ m luminosities of $>10^{12} L_{\odot}$ ) play a fundamental role in the cosmological evolution of galaxies and largescale structures. First discovered in significant numbers by the Infrared Astronomical Satellite (Soifer et al. 1984), they are almost invariably mergers (Farrah et al. 2001; Bushouse et al. 2002; Veilleux et al. 2002, 2006), powered by star formation and active galactic nucleus (AGN) activity, with the star formation usually dominating (Genzel et al. 1998; Veilleux et al. 1999; Rigopoulou et al. 1999; Imanishi et al. 2007; Vega et al. 2008). ULIRGs are rare at low redshift, with less than 50 at $z \lesssim 0.1$, but become much more numerous at high redshifts, reaching a density on the sky of several hundred per square degree at $z \gtrsim 1$ (Rowan-Robinson et al. 1997; Dole et al. 2001; Hughes et al. 1998; Barger et al. 1998; Borys et al. 2003; Mortier et al. 2005). The high-redshift ULIRGs appear similar in some ways to those in the local universe, in that many of them are starburst-dominated mergers (Farrah et al. 2002; Chapman et al. 2003; Smail et al. 2004; Takata et al. 2006; Borys et al. 2006; Valiante et al. 2007; Berta et al. 2007; Bridge et al. 2007; Lonsdale et al. 2009; Huang et al. 2009), though there are also signs of differences, e.g., a higher fraction of systems with no signs of interaction (Melbourne et al. 2008), systematically different mid-IR spectral shapes (Farrah et al. 2008), and overdense local environments (Blain et al. 2004; Farrah et al. 2006; Magliocchetti et al. 2007). Reviews of their properties can be found in Sanders \& Mirabel (1996) and Lonsdale et al. (2006).
The cosmological significance of ULIRGs makes a solid understanding of them at low redshifts important, but there remain several uncertainties over the lifecycle of low-redshift ULIRGs. We know that the merger activity triggers star formation and AGN activity, but we do not know how long the starburst lasts, whether or not there exist distinct "AGN plus starburst" or "AGN-dominated" phases, the fraction of ULIRGs that become quasars, or if there are multiple evolutionary paths that a ULIRG can take.

In this paper, we explore a new approach to study the evolution of the low-redshift ULIRG population. Since many of the best diagnostics of star formation and AGN activity lie in the mid-infrared, we start with a mid-infrared spectroscopic data set of $z<0.4$ ULIRGs, taken with the Infrared Spectrograph (IRS; Houck et al. 2004) on board the Spitzer Space Telescope (Werner et al. 2004; Soifer et al. 2008). To this data set, we apply two novel analysis methods to determine trends in mid-IR spectral shape across the sample. First, we present a Bayesian based estimator of the degree of similarity between a pair of ULIRG spectra. This estimator uses data from every resolution element, marginalizing over measurement error, luminosity, and foreground obscuration, to produce Bayes factors that describe the degree of resemblance between every possible pair of spectra. Second, we use methods developed using graph theory to study interconnected groups of entities to produce a "network" diagram that visualizes these Bayes factors across the whole sample simultaneously. We combine these results with archival data to propose an evolutionary description of ULIRGs in the low-redshift universe. 
This paper is structured as follows. In Section 2, we describe the sample selection and data, outline the method by which we calculate the Bayes factors, and describe the network construction. In Section 3, we present the Bayes factors and network diagram. In Section 4, we assess the robustness of the Bayes factors and the network diagram, and possible sources of error. Discussion can be found in Section 5. We summarize our conclusions in Section 6 . We assume a spatially flat cosmology with $H_{0}=70 \mathrm{~km} \mathrm{~s}^{-1} \mathrm{Mpc}^{-1}, \Omega=1$, and $\Omega_{m}=0.3$.

\section{METHOD}

\subsection{The Sample}

The sample was observed as part of the IRS Guaranteed Time program to obtain mid-infrared spectra of low-redshift IRluminous sources (Spitzer program ID 105), selected from the IRAS 1 Jy (Kim \& Sanders 1998) and 2 Jy (Strauss et al. 1990) surveys, and from the FIRST sample (Stanford et al. 2000). The sample is slightly biased toward sources with warm infrared colors, as described in Desai et al. (2007), but should still be representative of the low-redshift IR-luminous galaxy population. A few of our sample have IR luminosities that fall outside the usual definition of a ULIRG, but for simplicity we refer to all of our sample as ULIRGs for the remainder of this paper.

Low-resolution spectra $(5.2 \mu \mathrm{m}-38.5 \mu \mathrm{m}, R \sim 60-125)$ were obtained of 118 objects, and high-resolution spectra (9.6 $\mu \mathrm{m}-38.0 \mu \mathrm{m}, R \sim 600)$ were obtained of a subset of 53 objects. Data reduction methods and initial results are presented in Armus et al. (2004), Spoon et al. (2004), and Armus et al. (2006). Further results are presented in Higdon et al. (2006), Spoon et al. (2006, 2007), and Desai et al. (2007). Atlases of the high- and low-resolution spectra can be found in Farrah et al. (2007) and L. Armus et al. (2009, in preparation), respectively.

The high-resolution spectra contain more elements than the low-resolution spectra, but sky background cannot be subtracted from them as we lack dedicated contemporaneous sky observations. Therefore, we use the low-resolution spectra. We exclude objects with $z>0.4$, so that we see approximately the same wavelength range for all objects. We also exclude IRAS $11119+3257$ and IRAS $23365+3604$ as they have poor quality data in the short-low modules. This leaves 102 objects, listed in Table 1. We smooth the spectra to the instrumental resolution using a $0.4 \mu \mathrm{m}$ boxcar, and assume a $5 \%$ flux error for each resulting resolution element, consistent with the observed variations between individual nod positions.

\subsection{Analysis}

\subsubsection{Bayesian Measures of Resemblance}

To compute the level of resemblance between any pair of (rest-frame) spectra, we adopt a Bayesian approach. For any two spectra, A and B, we compute

$$
\mathcal{R}=\frac{P(\mathrm{~A}, \mathrm{~B} \mid \text { different })}{P(\mathrm{~A}, \mathrm{~B} \mid \text { same })},
$$

where $P(\mathrm{~A}, \mathrm{~B} \mid$ same $)$ is the probability density that the two spectra are identical and $P(\mathrm{~A}, \mathrm{~B} \mid$ different $)$ is the probability density that the spectra are different. The quantity $\mathcal{R}$ is thus the Bayes factor $^{7}$ (Jeffreys 1961; Connolly et al. 2006) quantifying

\footnotetext{
7 Arguably, the ratio of posteriors, i.e., $P($ different $\mid \mathrm{A}, \mathrm{B}) / P($ same $\mid \mathrm{A}, \mathrm{B})$ is a more intuitive statistic. However, the calculation of the odds requires one to make prior assumptions about $P$ (same) and $P$ (different), which we prefer to avoid.
}

the belief ${ }^{8}$ that this pair of spectra arises from sources whose physical properties (or at least those that give rise to the midIR emission) are the same. In essence, we are performing a pixel-by-pixel comparison between two spectra, where no spectral region is preferentially weighted. While this method could be used to compare data to models, we are here making no model comparisons, instead comparing the spectra to each other.

The simplest use of this method would involve computing $\mathcal{R}$ for every possible pair of "raw" (i.e., reduced, rest-frame but otherwise unaltered) spectra. This, however, is not enough. There exist two variables that will increase the $\mathcal{R}$ values, but which do not necessarily reflect physical differences. The first is instrument noise; differences between spectra that arise due to Gaussian fluctuations in the measurements should not contribute to the $\mathcal{R}$ values. The second is cold foreground extinction; if object A and object B are intrinsically identical, but object A has a thicker screen of cold dust in front of it, then this should not contribute to the $\mathcal{R}$ values either. We make a third, simplifying assumption that differences in mid-IR luminosity (i.e., multiplicative scalings between spectra) do not reflect "real" differences. Therefore, in calculating the $\mathcal{R}$ values, we marginalize with respect to instrument noise, mid-IR luminosity, and cold foreground extinction. The resulting (fully marginalized) probabilities, $P(\mathrm{~A}, \mathrm{~B} \mid$ same $)$ and $P(\mathrm{~A}, \mathrm{~B} \mid$ different $)$, are thus measures of the evidence for the hypothesis (Sivia 1996). The full methodology is described in the Appendix.

Finally, we adopt a boundary condition for $\log _{10}(\mathcal{R})$; pairs of spectra with $\log _{10}(\mathcal{R})$ below this boundary are treated as similar, and those pairs with $\log _{10}(\mathcal{R})$ above it are treated as different. We set this boundary at $\log _{10}(\mathcal{R})=0$. In frequentist terms, this boundary is equivalent to demanding $\chi^{2} \lesssim 0.8$ (Sellke et al. 2001). In Bayesian terms, using the scale given in Jeffreys (1961), this corresponds to "marginal" strength of evidence. We explore the sensitivity of our results to the error in $\mathcal{R}$ and choice of boundary condition in Section 4.

\subsubsection{Network Construction}

With a sample of 102 galaxies, we have ${ }^{102} C_{2}=5151 \mathcal{R}$ values, one for each possible pair. Our second requirement is a method to study these Bayes factors across the sample.

This is an example of studying a pairwise-connected group of entities. Other examples include a computer network (e.g., Siganos et al. 2003), predator-prey relationships among animals, or "social" networks such as friendships between individuals in a group. As such, a common terminology has arisen to describe them. Each entity (e.g., a computer or a person) is a "node." Connections between nodes are "edges" if they have no direction (for example, if two computers share data) and "arcs" if they have direction (for example, lions eat gazelles but gazelles do not eat lions). The number of edges connecting to a node is the "degree" of that node. The nodes in our network are the ULIRGs. The pairs where $\log _{10}(\mathcal{R})<0$ are connected via edges, while those pairs with $\log _{10}(\mathcal{R})>0$ are not connected.

Several methods have been developed to plot nodes and their connections in informative ways. In our case, we require a method that (1) places connected nodes close together and (2) produces as few "crossing" edges as possible. A suitable algorithm for this is a "force-directed" algorithm, in which

\footnotetext{
8 We use the word "belief" in its Bayesian sense, i.e., the odds of a successful trial of the truth of a given proposition, and not in the colloquial sense.
} 
Table 1

The Sample

\begin{tabular}{|c|c|c|c|c|c|c|}
\hline ID & Galaxy & R.A. (J2000) & Decl. & $z$ & Class $^{\mathrm{a}}$ & $L_{i r}{ }^{2}$ \\
\hline 1 & IRAS 05189-2524 & 052101.5 & -252145.4 & 0.043 & S2 & \\
\hline 2 & IRAS $08572+3915$ & 090025.4 & +390354.4 & 0.058 & $\mathrm{~L}$ & \\
\hline 3 & IRAS $12112+0305$ & 121346.0 & +024838.0 & 0.073 & $\mathrm{~L}$ & \\
\hline 4 & IRAS $14348-1447$ & 143738.4 & -150022.8 & 0.083 & $\mathrm{~L}$ & \\
\hline 5 & IRAS $15250+3609$ & 152659.4 & +355837.5 & 0.055 & $\mathrm{~L}$ & \\
\hline 6 & IRAS 22491-1808 & 225149.3 & -175223.5 & 0.078 & $\mathrm{H}$ & \\
\hline 7 & Arp 220 & 153457.1 & +233011.5 & 0.018 & $\mathrm{~L}$ & \\
\hline 8 & Mrk 231 & 125614.2 & +565225.2 & 0.042 & S1 & \\
\hline 9 & Mrk 273 & 134442.1 & +555312.7 & 0.038 & S2 & \\
\hline 10 & UGC 5101 & 093551.7 & +612111.3 & 0.039 & $\mathrm{~L}^{\mathrm{d}}$ & \\
\hline 11 & IRAS F00183-7111 & 002034.7 & -705526.7 & 0.327 & $\mathrm{~L}^{\mathrm{e}}$ & \\
\hline 12 & IRAS F00188-0856 & 002126.5 & -083926.3 & 0.128 & $\mathrm{~L}$ & \\
\hline 13 & IRAS $00199-7426$ & 002207.0 & -740941.7 & 0.096 & $H ?^{f}$ & \\
\hline 14 & IRAS $00275-0044$ & 003009.1 & -002744.2 & 0.242 & ? & \\
\hline 15 & IRAS 00275-2859 & 003004.2 & -284225.0 & 0.278 & $\mathrm{~S} 1^{\mathrm{g}}$ & \\
\hline 16 & IRAS 00397-1312 & 004215.5 & -125602.8 & 0.262 & $\mathrm{H}$ & \\
\hline 17 & IRAS $00406-3127$ & 004303.2 & -311049.5 & 0.342 & $\mathrm{~S}^{\mathrm{h}}$ & \\
\hline 18 & IRAS 01003-2238 & 010250.0 & -222157.5 & 0.118 & $\mathrm{H}$ & \\
\hline 19 & IRAS $01199-2307$ & 012220.9 & -225206.7 & 0.156 & $\mathrm{H}$ & \\
\hline 20 & IRAS $01298-0744$ & 013221.4 & -072908.1 & 0.136 & $\mathrm{H}$ & \\
\hline 21 & IRAS $01355-1814$ & 013757.4 & -175920.6 & 0.192 & $\mathrm{H}$ & \\
\hline 22 & IRAS $01388-4618$ & 014055.9 & -460253.6 & 0.090 & $\mathrm{H}^{\mathrm{i}}$ & \\
\hline 23 & IRAS 01494-1845 & 015151.4 & -183046.4 & 0.152 & $\mathrm{H}^{\mathrm{h}}$ & \\
\hline 24 & IRAS $02054+0835$ & 020806.8 & +085002.0 & 0.345 & $\mathrm{~S} 1^{\mathrm{g}}$ & \\
\hline 25 & IRAS 02113-2937 & 021333.0 & -292339.5 & 0.192 & $\mathrm{~L}^{\mathrm{h}}$ & \\
\hline 26 & IRAS F02115+0226 & 021410.3 & +023959.7 & 0.399 & ? & \\
\hline 27 & IRAS F02455-2220 & 024751.3 & -220737.8 & 0.296 & ? & \\
\hline 28 & IRAS 02530+0211 & 025534.4 & +02 2341.4 & 0.028 & $\mathrm{~L}^{\mathrm{i}}$ & \\
\hline 29 & IRAS 03000-2719 & 030211.4 & -270726.3 & 0.221 & ? & \\
\hline 30 & IRAS $03158+4227$ & 031912.4 & +423828.0 & 0.134 & $L ?^{j}$ & \\
\hline 31 & IRAS $03521+0028$ & 035442.1 & +00 3703.4 & 0.152 & $\mathrm{~L}^{\mathrm{k}}$ & \\
\hline 32 & IRAS $03538-6432$ & 035425.2 & -642344.7 & 0.301 & ? & \\
\hline 33 & IRAS 04114-5117 & 041244.2 & -510940.8 & 0.125 & $?$ & \\
\hline 34 & IRAS 04313-1649 & 043337.1 & -164331.5 & 0.268 & $\mathrm{~L}^{\mathrm{i}}$ & \\
\hline 35 & IRAS 04384-4848 & 043950.8 & -484317.4 & 0.203 & $\mathrm{H}^{1}$ & \\
\hline 36 & IRAS 06009-7716 & 055837.1 & -771639.0 & 0.117 & $\mathrm{H}$ ? & \\
\hline 37 & IRAS $06035-7102$ & 060254.0 & $\begin{array}{llll}-71 & 03 & 10.2\end{array}$ & 0.079 & $\mathrm{H}^{\mathrm{f}}$ & \\
\hline 38 & IRAS 06206-6315 & 062101.2 & -631723.5 & 0.092 & $\mathrm{~S} 2^{\mathrm{f}}$ & \\
\hline 39 & IRAS 06301-7934 & 062642.5 & -793631.0 & 0.156 & ? & \\
\hline 40 & IRAS $06361-6217$ & 063635.8 & -622033.1 & 0.160 & $\mathrm{~L}^{\mathrm{e}}$ & \\
\hline 41 & IRAS 07145-2914 & 071631.2 & -291928.8 & 0.006 & $\mathrm{~S} 2^{\mathrm{m}}$ & \\
\hline 42 & IRAS $07449+3350$ & 074810.6 & +334327.1 & 0.355 & $\mathrm{~L}^{\mathrm{n}}$ & \\
\hline 43 & IRAS $07598+6508$ & 080433.1 & +645948.6 & 0.148 & S1 & \\
\hline 44 & IRAS F08208+3211 & 082354.6 & +320212.0 & 0.396 & $\mathrm{H}^{\circ}$ & \\
\hline 45 & IRAS $08559+1053$ & 085841.8 & +10 4121.9 & 0.148 & S2 & \\
\hline 46 & IRAS 09022-3615 & 090412.7 & -362701.1 & 0.060 & ? & \\
\hline 47 & IRAS 09463+8141 & 095300.5 & +812728.4 & 0.155 & $\mathrm{~L}$ & \\
\hline 48 & IRAS $10091+4704$ & 101216.7 & +464943.5 & 0.246 & $\mathrm{~L}$ & \\
\hline 49 & IRAS $10378+1109$ & 104029.2 & +105318.3 & 0.136 & $\mathrm{~L}$ & \\
\hline 50 & IRAS $10565+2448$ & 105918.1 & +243234.3 & 0.043 & $\mathrm{H}^{\mathrm{o}}$ & \\
\hline 51 & IRAS F11038+3217 & 110635.7 & +320146.4 & $0.130^{\mathrm{p}}$ & ? & \\
\hline 52 & IRAS $11095-0238$ & 111203.4 & +020422.4 & 0.107 & $\mathrm{~L}$ & \\
\hline 53 & IRAS $11223-1244$ & 112450.1 & -130113.5 & 0.199 & S2 & \\
\hline
\end{tabular}

$4,21,25,27,29,33,35,39,44,58,66,72,76,78,82,83,87,89,92,93$

$3,9,19,21,26,27,29,33,35,38,49,51,53,58,60,66,72,78,82,83,86,87,89,92,93,94$

$19,22,26,30,35,38,52,53,56,58,67,70,74$

$$
7,21,31,93
$$

$11,15,17,24,32,45,62,69,81,84,95$

$4,13,19,22,23,25,26,27,29,32,35,38,42,49,51,52,53,54,56,58,60,65,66,67,70,71,72,74,82,83,85,86,89,94$ $12,14,37,40,45,95$

$$
8,37
$$

$10,37,40,53,70,80$

$9,22,23,26,29,32,36,42,50,51,56,60,67,70,72,74$

$10,36,37,46,63$

$8,59,84,90,95,99$

$1,8,28,74,80,88,91$

$1,28,55,57,64,68,75,91$

$4,5,9,21,30,35,52,56,86,89,92$ $51,54,71$
$3,4,6,7,19,93$

12.35
12.44

2.05

12.05
12.27
13.08

3.08

12.82

2.74

11.10
12.59

12.48

12.55

2.73

12.59

2.36

12.03
12.19

12.17

2.33

10.08

2.84

12.46

2.28

12.26
12.24

2.61

12.35
12.01

1.45

11.45
12.29

$5,9,13,23,26,29,35,38,50,51,52,56,58,60,68,70,72,74,77,88,9$

$9,13,22,25,26,29,36,42,44,50,51,60,70,72$

$$
8,62,99
$$

$3,9,23,29,36,44,51,60,70,72,73,85$

$1,4,5,9,13,22,23,27,29,32,35,38,42,44,50,51,53,54,56,58,60,67,70,72,88$ $3,4,9,26,29,42,44,51,60,72$ $17,18,57,75$

$3,4,9,13,22,23,25,26,27,35,38,42,44,50,51,53,60,67,70,72,74,80,83$ $5,19,35,49,52$

$$
6,7,87
$$

$1,8,9,13,26,37,38,40,42,46,51,53,63,67,69,70,80,88$

$$
3,4,44,47,76,87
$$

$3,4,5,9,19,22,26,29,30,38,44,50,52,53,54,56,58,60,70,72,74,80,86,88,89$ $13,14,23,25,42,60,72,73,85$

$10,11,12,14,32,40,45,46,5$

$4,5,9,22,26,29,32,35,42,44,51,52,53,54,56,58,60,67,70,71,72,74,80,82,86,89$ 3,7

$10,12,32,37,42,46,63$

$1,9,13,23,26,27,29,32,36,38,40,44,46,51,67,70,72,78,85$ $59,61,90$

$3,23,25,26,27,29,33,35,38,42,53,72,76,83,85$ $8,10,37,46,84,95$

$14,32,37,40,42,45,62,63,67,69,70,73,95$ $33,71,73,76$

$$
4,9,30,94
$$

$13,22,23,26,29,35,51,56,58,60,67,68,72,74,77,91$

$4,9,13,20,22,23,25,26,27,29,32,37,38,42,50,53,56,70,71,72,74,80,85$

$5,9,19,22,30,35,38,54,56,58,70,72,74,80,86,88$

$4,5,9,12,26,29,32,35,38,44,51,60,67,72,78$ 
Table 1

(Continued)

\begin{tabular}{|c|c|c|c|c|c|c|c|}
\hline ID & Galaxy & R.A. (J2000) & Decl. & $z$ & Class $^{\mathrm{a}}$ & $L_{i r}{ }^{\mathrm{b}}$ & Connections $^{\mathrm{c}}$ \\
\hline 54 & IRAS $11582+3020$ & 120046.8 & +300414.8 & 0.223 & $\mathrm{~L}$ & 12.55 & $9,20,26,35,38,52,56,74,80,88,92$ \\
\hline 55 & IRAS 12018+1941 & 120424.5 & +192510.3 & 0.169 & $\mathrm{~L}$ & 12.54 & 18,57 \\
\hline 56 & IRAS $12032+1707$ & 120547.7 & +165108.0 & 0.217 & $\mathrm{~L}^{\mathrm{k}}$ & 12.59 & $5,9,13,19,22,26,35,38,50,51,52,54,58,60,70,74,80,88,92$ \\
\hline 57 & IRAS 12071-0444 & 120945.1 & -050113.9 & 0.128 & S2 & 12.44 & $1,18,28,55,64,68,75$ \\
\hline 58 & IRAS $12205+3356$ & 122300.3 & +333928.9 & 0.263 & ? & 12.49 & $3,4,5,9,22,26,35,38,50,52,56,60,66,72,82,83$ \\
\hline 59 & IRAS $12514+1027$ & 125400.8 & +1011 12.4 & 0.319 & $\mathrm{~S} 2^{\mathrm{g}}$ & 12.72 & $2,15,43,90$ \\
\hline 60 & IRAS $13120-5453$ & 131506.4 & -550922.7 & 0.031 & S2? & 12.26 & $4,9,13,22,23,25,26,27,29,35,36,38,50,53,56,58,65,66,67,70,72,74,78,82,83,89,94$ \\
\hline 61 & IRAS $13218+0552$ & 132419.9 & +053704.7 & 0.205 & S1 & 12.73 & 43 \\
\hline 62 & IRAS $13342+3932$ & 133624.1 & +391731.1 & 0.179 & S1 & 12.47 & $1,8,24,46,81,84$ \\
\hline 63 & IRAS $13352+6402$ & 133650.7 & +634703.0 & 0.237 & ? & 12.50 & $14,32,37,40,46,85$ \\
\hline 64 & IRAS $13451+1232$ & 134733.3 & +12 1724.2 & 0.121 & S2 & 12.37 & $1,18,57,75,97$ \\
\hline 65 & IRAS $14070+0525$ & 140931.3 & +05 1131.8 & 0.264 & S2 & 12.88 & $9,60,71,73$ \\
\hline 66 & IRAS $14378-3651$ & 144059.0 & -370432.0 & 0.068 & $L^{f}$ & 12.07 & $3,4,9,58,60,72,82,83,87,89,93,94$ \\
\hline 67 & IRAS $15001+1433$ & 150231.9 & +142135.1 & 0.163 & S2 & 12.48 & $1,5,9,13,26,29,32,37,38,42,46,50,53,60,68,69,70,72,74,80,88,91,97$ \\
\hline 68 & IRAS $15206+3342$ & 152238.0 & +333135.9 & 0.124 & $\mathrm{H}$ & 12.27 & $1,18,22,50,57,67,69,74,75,88,91,97$ \\
\hline 69 & IRAS $15462-0450$ & 154856.8 & -045933.6 & 0.100 & S1 & 12.24 & $1,8,32,37,46,67,68,88,97$ \\
\hline 70 & IRAS $16090-0139$ & 161140.5 & -014705.6 & 0.134 & $\mathrm{~L}$ & 12.58 & $5,9,12,13,22,23,25,26,29,32,35,38,42,46,51,52,56,60,67,71,72,73,74,78,80$ \\
\hline 71 & IRAS $16300+1558$ & 163221.4 & +155145.2 & 0.242 & $\mathrm{~L}$ & 12.69 & $9,20,38,47,51,65,70,73$ \\
\hline 72 & IRAS $16334+4630$ & 163452.6 & +462452.8 & 0.191 & $\mathrm{~L}$ & 12.41 & $3,4,9,13,22,23,25,26,27,29,35,36,38,42,44,50,51,52,53,58,60,66,67,70,74,78,82,83,85,89$ \\
\hline 73 & IRAS F16576+3553 & 165924.7 & +354901.7 & 0.371 & $\mathrm{~L}^{\mathrm{n}}$ & 12.39 & $25,36,46,47,65,70,71,85$ \\
\hline 74 & IRAS $17068+4027$ & 170832.1 & +402328.2 & 0.179 & $\mathrm{H}$ & 12.33 & $5,9,13,17,22,29,35,38,50,51,52,54,56,60,67,68,70,72,77,80,88$ \\
\hline 75 & IRAS 17179+5444 & 171854.2 & +544147.3 & 0.147 & S2 & 12.30 & $1,18,28,57,64,68,91,97$ \\
\hline 76 & IRAS $17208-0014$ & 172322.0 & -001700.9 & 0.043 & $\mathrm{H}^{\mathrm{d}}$ & 11.94 & $3,33,44,47,83,87,89$ \\
\hline 77 & IRAS F17252+3659 & 172657.8 & +365639.5 & 0.365 & ? & 12.47 & $22,50,74,91$ \\
\hline 78 & IRAS $17463+5806$ & 174705.6 & +580518.0 & 0.309 & $\mathrm{~S} 2 \mathrm{q}$ & 12.61 & $3,4,42,53,60,70,72$ \\
\hline 79 & IRAS $18030+0705$ & 180527.1 & +0705 57.5 & 0.146 & ? & 12.16 & ... \\
\hline 80 & IRAS $18443+7433$ & 184254.8 & +743621.0 & 0.135 & ? & 12.27 & $12,17,29,32,35,38,51,52,54,56,67,70,74,88$ \\
\hline 81 & IRAS $19254-7245 \mathrm{~S}$ & 193121.6 & -723922.0 & 0.063 & $\mathrm{~S} 2^{\mathrm{r}}$ & 12.19 & $1,8,62,97$ \\
\hline 82 & IRAS 19297-0406 & 193221.3 & -035956.3 & 0.086 & $\mathrm{H}^{\mathrm{d}}$ & 12.37 & $3,4,9,38,58,60,66,72,83,86,87,89,92,93,94$ \\
\hline 83 & IRAS 19458+0944 & 194815.7 & +095205.0 & 0.100 & ? & 12.34 & $3,4,9,29,44,58,60,66,72,76,82,87,89,92$ \\
\hline 84 & IRAS $20037-1547$ & 200631.7 & -153908.0 & 0.192 & $\mathrm{~S} 1^{\mathrm{g}}$ & 12.52 & $8,15,37,45,62,95$ \\
\hline 85 & IRAS $20087-0308$ & 201123.9 & -025950.7 & 0.106 & $L^{f}$ & 12.34 & $9,25,36,42,44,51,63,72,73$ \\
\hline 86 & IRAS 20100-4156 & 201329.5 & -414734.9 & 0.130 & $\mathrm{H}^{\mathrm{f}}$ & 12.52 & $4,9,19,35,38,52,82,89,92$ \\
\hline 87 & IRAS 20414-1651 & 204418.2 & -164016.2 & 0.087 & $\mathrm{H}$ & 12.18 & $3,4,31,33,66,76,82,83,89$ \\
\hline 88 & IRAS 20551-4250 & 205826.8 & -423900.3 & 0.043 & $\mathrm{H}^{\mathrm{f}}$ & 12.00 & $17,22,26,32,35,52,54,56,67,68,69,74,80,91$ \\
\hline 89 & IRAS $21272+2514$ & 212929.4 & +252750.0 & 0.151 & $\mathrm{~S} 2^{\mathrm{k}}$ & 12.10 & $3,4,9,19,35,38,60,66,72,76,82,83,86,87,92,93$ \\
\hline 90 & IRAS $23060+0505$ & 230833.9 & +052129.9 & 0.173 & S2 & 12.55 & $15,43,59,98,99,102$ \\
\hline 91 & IRAS $23128-5919$ & 231546.8 & -590315.6 & 0.045 & $\mathrm{H}^{\mathrm{f}}$ & 11.97 & $1,17,18,22,50,67,68,75,77,88$ \\
\hline 92 & IRAS $23129+2548$ & 231521.4 & +260432.2 & 0.179 & $\mathrm{~L}^{\mathrm{k}}$ & 12.43 & $3,4,19,54,56,82,83,86,89$ \\
\hline 93 & IRAS $23230-6926$ & 232603.6 & -691018.8 & 0.106 & $L^{f}$ & 12.25 & $3,4,6,21,66,82,89$ \\
\hline 94 & IRAS $23253-5415$ & 232806.1 & -535831.0 & 0.130 & $\mathrm{H}^{\mathrm{s}}$ & 12.37 & $4,9,49,60,66,82$ \\
\hline 95 & IRAS $23498+2423$ & 235226.0 & +24 4016.7 & 0.212 & S2 & 12.51 & $8,10,15,37,45,46,84$ \\
\hline 96 & 3C 273 & 122906.7 & +020308.6 & 0.158 & $\mathrm{~S} 1^{\mathrm{g}}$ & 12.83 & 100,102 \\
\hline 97 & Mrk 1014 & 015950.2 & +002340.6 & 0.163 & $\mathrm{~S} 1^{\mathrm{g}}$ & 12.63 & $1,64,67,68,69,75,81$ \\
\hline 98 & Mrk 463 & 135602.9 & +18 2219.1 & 0.050 & $\mathrm{~S} 2^{\mathrm{g}}$ & 11.80 & $90,99,100,101,102$ \\
\hline
\end{tabular}


Table 1

(Continued)

\begin{tabular}{|c|c|c|c|c|c|c|c|}
\hline ID & Galaxy & R.A. (J2000) & Decl. & $z$ & $\begin{array}{c}\text { Class }^{\mathrm{a}} \\
\end{array}$ & 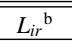 & "Connections ${ }^{\mathrm{c}}$ \\
\hline 99 & PG $1119+120$ & 112147.1 & +114418.3 & 0.050 & $\mathrm{~S} 1^{\mathrm{g}}$ & 11.29 & $15,24,90,98,101,102$ \\
\hline 100 & PG $1211+143$ & 121417.7 & +140312.6 & 0.081 & $\mathrm{~S} 1^{g}$ & 11.76 & $96,98,102$ \\
\hline 101 & PG $1351+640$ & 135315.8 & +634545.4 & 0.088 & $\mathrm{~S} 1^{\mathrm{g}}$ & 11.88 & 98,99 \\
\hline 102 & PG 2130+099 & 213227.8 & +1008 19.5 & 0.063 & $\mathrm{~S} 1^{\mathrm{g}}$ & 11.60 & $90,96,98,99,100$ \\
\hline
\end{tabular}

Notes.

${ }^{a}$ Optical spectral classification, taken from Veilleux et al. (1999) unless otherwise indicated. These vary significantly in reliability; see references for details.

${ }^{\mathrm{b}}$ Infrared luminosities are either taken from Farrah et al. (2003), or calculated from the IRAS fluxes using the same methods. Units are the logarithm of the rest-frame $1-1000 \mu \mathrm{m}$ luminosity, in solar luminosities $(3.826 \times$ $\left.10^{26} \mathrm{~W}\right)$.

ID numbers of the ULIRGs that "pair" with this object, i.e., that have $\log (\mathcal{R})<0$.

d Veilleux et al. (1995)

${ }^{\mathrm{e}}$ Armus et al. (1989).

${ }^{\mathrm{f}}$ Duc et al. (1997).

g Véron-Cetty \& Véron (2001).

${ }^{\mathrm{h}}$ Allen et al. (1991).

Kewley et al. (2001).

Meusinger et al. (2001).

${ }^{k}$ Darling \& Giovanelli (2006).

${ }^{1}$ Lawrence et al. (1999).

Bergvall et al. (1986)

Stanford et al. (2000).

Rupke et al. (2005).

Measured from the IRS spectrum. The redshift given by Stanford et al. (2000) does not agree with the IRS data.

Leech et al. (1994).

Mirabel et al. (1991).

Sekiguchi \& Wolstencroft (1993). 

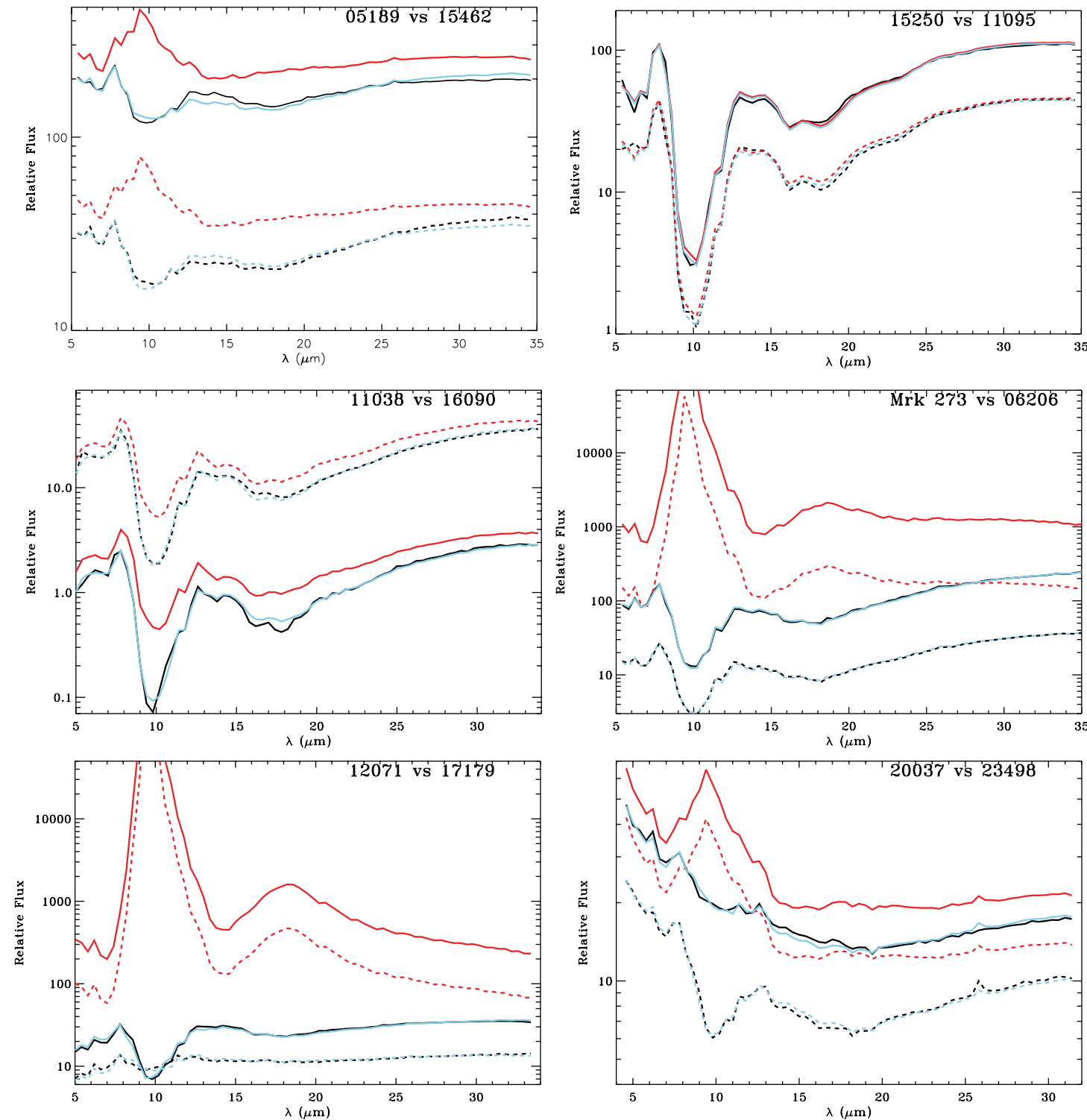

Figure 1. Six examples of the "best" fits (for which $-150<\log (\mathcal{R})<-250$ ), calculated by minimizing Equation (A31). The solid (dashed) black line is the IRS spectrum for object A (B). The solid (dashed) red line is the predicted intrinsic spectrum (i.e., without foreground extinction) for object A (B). The solid (dashed) blue line is the predicted intrinsic spectrum with cold foreground extinction applied for object A (B). The identical shapes of the solid and dashed red lines show that the intrinsic spectra of objects A and B are the same (they differ by a factor of $\left.f e^{f_{e} b_{e}} /(1-f) e^{\left(1-f_{e}\right) b_{e}}\right)$. The identical shapes of the solid (dashed) black and blue lines show that the observed and "predicted observed" spectra of object A (B) are the same. We do not constrain the shape of the intrinsic spectrum, and are showing the purely mathematically "best" fits, so some predicted features in the intrinsic spectra, such as the very strong silicate emission feature in the bottom left panel, may not be "real."

(A color version of this figure is available in the online journal.)

attractive and repulsive forces govern the arrangement of the nodes and edges (Kamada \& Kawai 1989; Fruchterman \& Reingold 1991). Connected nodes attract each other along edges, while all nodes repel each other. The attractive force is modeled as if the edges are springs (i.e., a Hookes law-type force) while the repulsive force is modeled as if the nodes are electrically charged (i.e., a Coulomb-type force). The parameters of the two forces are adjusted, and nodes allowed to move according to the forces acting on them until (1) an equilibrium state is reached in which the positions of the nodes and edges do not change appreciably and (2) the nodes and edges can be seen simultaneously.

To create the network for our sample, we use two software packages; the Network Workbench tool, ${ }^{9}$ and Cytoscape. ${ }^{10}$

\footnotetext{
9 This tool is developed jointly by Indiana University and Northwestern University, and is available from http://nwb.slis.indiana.edu.
}

10 Available from http://cytoscape.org/.

\section{RESULTS}

The pairs of sources for which $\log _{10}(\mathcal{R})<0$ are given in Table 1. Examples of pairs where $\log _{10}(\mathcal{R})<0$ are shown in Figure 1, and examples of pairs with $\log _{10}(\mathcal{R})>0$ are shown in Figure 2. Presenting all of the $\mathcal{R}$ values would take an unreasonable amount of space, so instead we plot their histogram in Figure 3. The adjacency matrix, $A$, for our network $^{11}$ is too large to give in full, but the first few elements of it read

$$
A=\left[\begin{array}{llll}
0 & 0 & 0 & 0 \\
0 & 0 & 0 & 0 \\
0 & 0 & 0 & 1 \\
0 & 0 & 1 & 0
\end{array}\right],
$$

\footnotetext{
11 The adjacency matrix, $A$, for an undirected graph with $n$ nodes is defined as the $n \times n$ matrix, where $A_{i j}$ is the number of edges from vertex $i$ to vertex $j$ and $A_{i i}$ is the number of loops for vertex $i$
} 

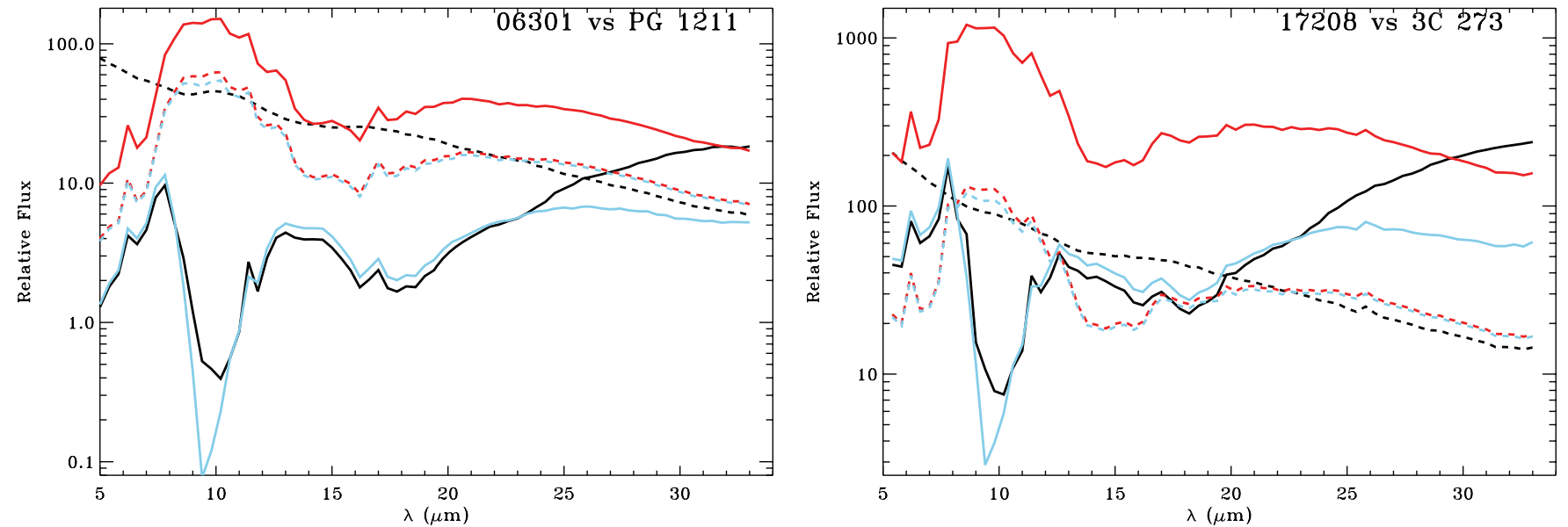

Figure 2. For comparison with Figure 1: two examples of the "worst" fits calculated by minimizing Equation (A31), where $\log (\mathcal{R}) \simeq 3000$. Here, the solid and dashed red lines are identical, but the blue and black lines are completely different for both objects, showing that the IRS spectrum and the "predicted" IRS spectrum do not match each other.

(A color version of this figure is available in the online journal.)

where the diagonal elements are zero as our network contains no loops. The network for the sample is shown in Figure 4.

\subsection{Structure}

Figure 4 exhibits a strong degree of connectivity. Only four objects $(16,41,48,79$, which are not plotted) are not connected to any other. All the other nodes are connected to at least one other node, with most having three or more connections. The average node degree is high, at 10.2, and the average shortest path between any two nodes is short for a 102 node network, at 3.2 edges.

There is significant variation in node degree across the diagram (Figure 5). We quantify this by computing the $k$-nearest-neighbor distribution (Pastor-Satorras et al. 2001) for our network. We find that, as the average degree per node, $k_{\text {mean }}$, increases, so does $k_{N N} ; k_{N N} \simeq 0.5$ for $k_{\text {mean }} \simeq 3$, rising to $k_{N N} \simeq 1.2$ for $k_{\text {mean }} \simeq 15$. So, with the caveat of the small number of nodes, Figure 5 shows a correlation between the degree of a node and that of its neighbors-nodes with a high degree are more likely to be connected to other nodes with a high degree — and is thus an "assortative" network.

We identify at least two substructures. The first is a strongly interconnected group centered on object 29 (IRAS 03000-2919), accompanied by some outliers on the left-hand side, containing $\sim 60 \%$ of the sample. We call this group A. The second is a weakly interconnected group extending in the rightward direction from group A, and containing the remaining $40 \%$ of the sample. It is plausible, given the two "branches" in this second group, that it is composed of two groups: one extending along the top of Figure 4 and centered on object 15 (IRAS 00275-2859), and one on the lower side of Figure 4, centered on object 97 (Mrk 1014). We label these two branches groups B and C, respectively. Solely with this diagram to go on, however, this subdivision is tentative, as the purported group $\mathrm{C}$ resembles the "outliers" on the left side of group A. We discuss the robustness of this subdivision in Section 5.1.

\subsection{Node Properties}

In studies of networks, insight can be gained by coding the nodes according to some property of the nodes (for example, coding the networks in Lusseau \& Newman (2004) by gender

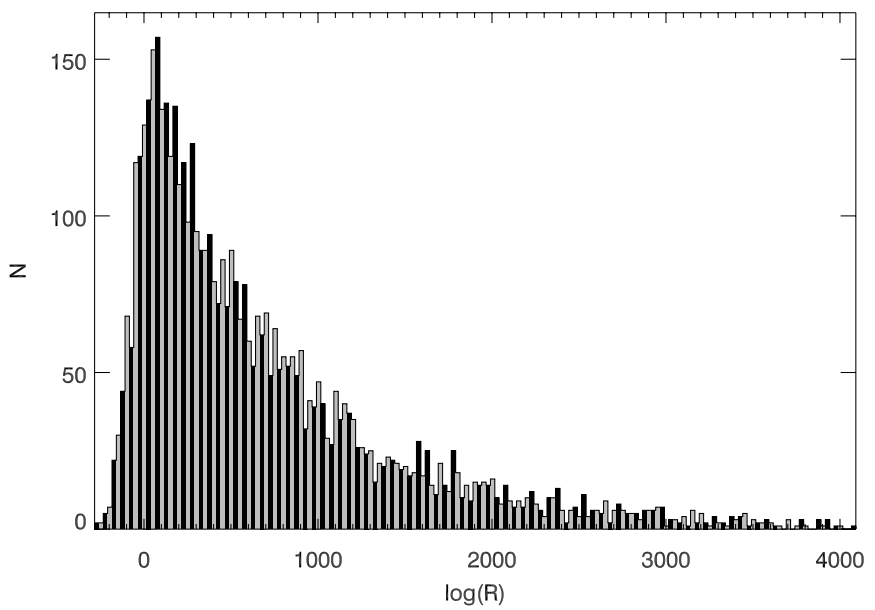

Figure 3. Histogram of the Bayes factors for every possible pair of spectra (5151 in total) in Table 1, computed using the miser algorithm.

or age revealed clear subcommunities). We adopt this approach in this section. We here present the individual coded diagrams, and interpret them in Section 5.

Optical spectral type. (Figure 6) The majority of the objects in group A have $\mathrm{H}$ II or LINER optical spectra, with a few Sy2's. This pattern is reversed in group B: most of the objects have Sy2 or Sy1 spectra with a small number of LINERs and $\mathrm{H}$ II's, especially toward the right-hand side where nearly all the objects are Syl's. In group C, there appear to be approximately equal numbers of all spectral types.

IR luminosity. (Figure 6) We expect a weakened correlation with $1-1000 \mu \mathrm{m}$ luminosity, given the large uncertainties caused by the paucity of flux measurements at $>100 \mu \mathrm{m}$. This expectation appears to be borne out; ${ }^{12}$ low- and high-luminosity systems are found in all three groups in approximately equal numbers, and there are no clear trends. There are no high-luminosity systems in group $\mathrm{C}$, though this may be due to the small number of objects in this group.

\footnotetext{
12 A weakening in the correlation should not arise from the marginalization over IR luminosity. Marginalizing over luminosity will remove any dependence on luminosity. However, if luminosity itself depends on (say) spectral shape at long wavelengths then the dependence on luminosity will remain, since we have not marginalized over spectral shape at long wavelengths.
} 


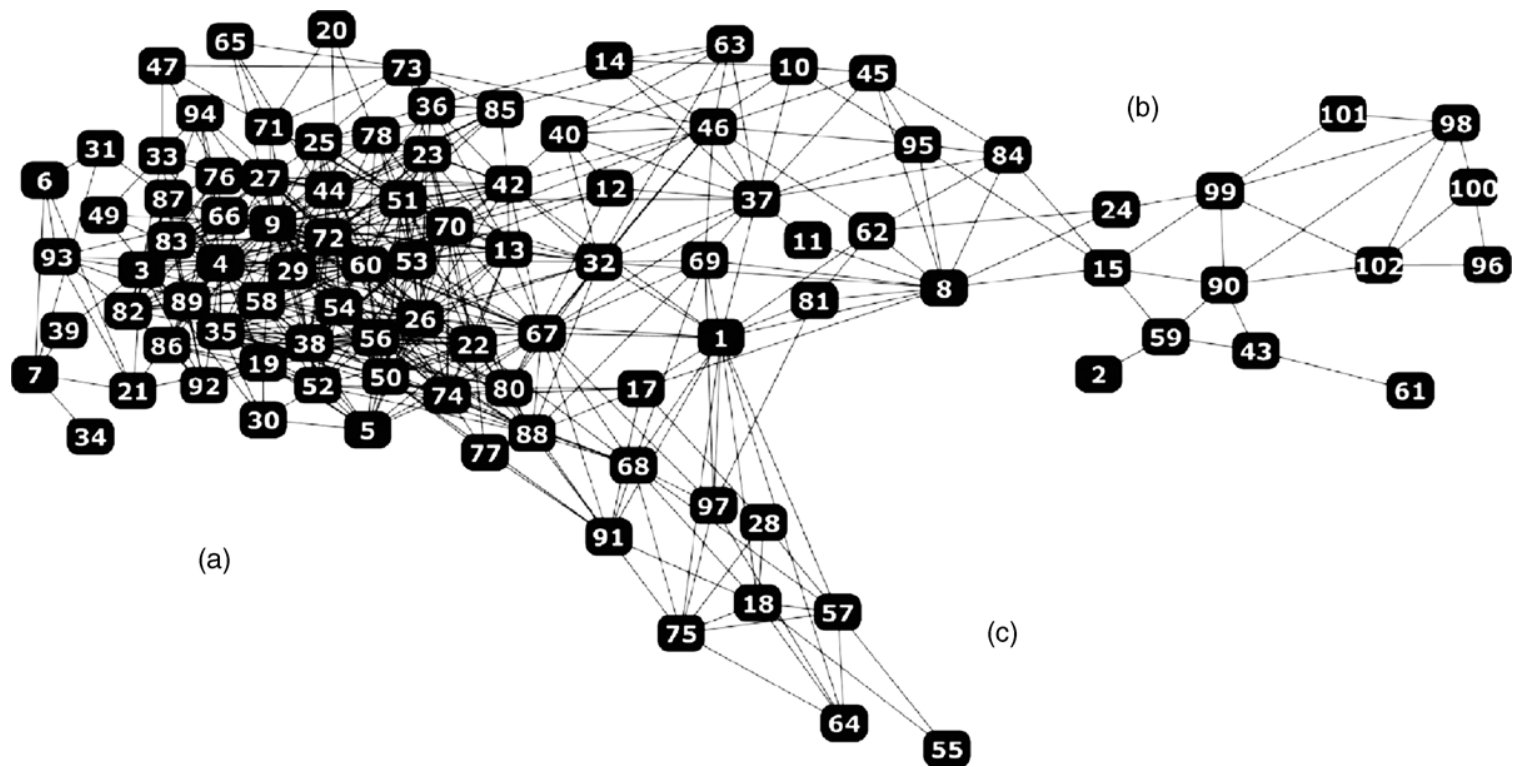

Figure 4. The network for our sample (generated using a spring-embedded algorithm within Cytoscape). The numbered points, or "nodes," are the objects in Table 1. An edge between two nodes indicates that $\log (\mathcal{R})<0$ for that pair of objects.

Projected nuclear separation. ${ }^{13}$ (Figure 7) There are strong caveats in interpreting this network; the imaging is heterogeneous (e.g., ground based for some, space based for others), the separations are projected rather than real, premergers that are widely separated can be erroneously identified as single nucleus systems and vice versa, and nuclear separations are degenerate with merger stage (Barnes \& Hernquist 1992; Dubinski et al. 1999). The figure does, however, show trends. While the single nucleus systems are found in all groups, the majority of them are in group B, including nearly all the objects at the end. Conversely, the widely and moderately separated systems are found almost exclusively in group A, with a few in groups B and C. Black hole mass. ${ }^{14}$ (Figure 7) Here, we use only those black hole (BH) masses measured via velocity dispersions (see, e.g., Tremaine et al. 2002), but the caveats are even stronger than for the nuclear separations diagram. Only 33 measurements are available, the random uncertainties are large, and the measurements depend critically on the calibration of the $\mathrm{M}_{\mathrm{BH}}{ }^{-}$ $M_{\text {bulge }}$ relation. Most importantly, the quantity that we are really interested in is not $M_{\mathrm{BH}}$, but rather the increase in $\mathrm{BH}$ mass during the merger (i.e., $\left.\Delta M_{\mathrm{BH}}\right|_{\text {merger }}$ ). The instantaneous snapshots of BH mass are of limited use since the BHs in the progenitors can in principle have a wide range of starting values. Therefore, this diagram is of limited interest. The intermediate mass BHs are spread randomly through the diagram, but $4 / 5$ high-mass BHs are at the end of group B, and 11/16 low-mass $\mathrm{BHs}$ are in group $\mathrm{A}$, with the rest lying in the first part of group $\mathrm{B}$, or group $\mathrm{C}$.

PAH equivalent width. (Figure 8) The mid-IR spectra of many ULIRGs show broad emission features at $6.2 \mu \mathrm{m}, 7.7 \mu \mathrm{m}$, $8.6 \mu \mathrm{m}, 11.2 \mu \mathrm{m}$, and $12.7 \mu \mathrm{m}$, attributed to bending and stretching modes in neutral and ionized polycyclic aromatic hydrocarbon (PAH) molecules, and it is now accepted that these features indicate ongoing star formation. Therefore, the

\footnotetext{
13 Taken from Rigopoulou et al. (1999), Farrah et al. (2001), Meusinger et al. (2001), Cui et al. (2001), Bushouse et al. (2002), Veilleux et al. (2002),

Veilleux et al. (2006), and Bianchi et al. (2008) and rescaled to our cosmology where appropriate.

14 Taken from Dasyra et al. (2006a, 2006b) and Kawakatu et al. (2007) and rescaled to our cosmology where appropriate.
}

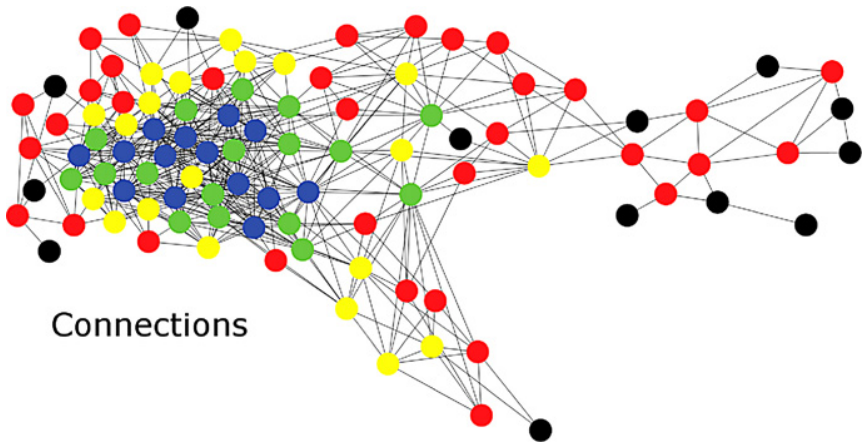

Figure 5. Network diagram, with the nodes color-coded by the number of edges connecting them (black $<4$, red $=4-7$, yellow $=7-14$, green $=14-20$, blue $>20)$

(A color version of this figure is available in the online journal.)

prominence of PAH features above the continuum, which we quantify via equivalent width, is a crude but reliable measure of the energetic importance of star formation ${ }^{15}$ (see also Genzel et al. 1998; Rigopoulou et al. 1999; Armus et al. 2006; Desai et al. 2007). As there is still debate over the use of individual PAH features as star formation rate diagnostics, we show networks coded by the $6.2 \mu \mathrm{m}$ and $11.2 \mu \mathrm{m}$ PAH features. The $11.2 \mu \mathrm{m}$ diagram is particularly striking. All the objects in group A have prominent PAHs and there is a high degree of homogeneity in their strengths. The PAHs then decline in prominence as we move left to right through groups B and C, until we reach the right-hand side of both groups where the PAHs are negligible. The $6.2 \mu \mathrm{m}$ diagram shows the same trends, but less obviously: most of the objects in group A have prominent PAHs with some outlying objects showing weakened features, and there is a less pronounced, though still clear, decline in PAH strength as we move left to right through groups B and C.

Silicate strength. (Figure 9, defined in Spoon et al. (2007) and Sirocky et al. (2008)). The $9.7 \mu \mathrm{m}$ feature is thought to arise from large silicate dust grains: in absorption when "cold" silicate

\footnotetext{
15 As opposed to PAH fluxes, which measure the absolute luminosity of the starburst.
} 

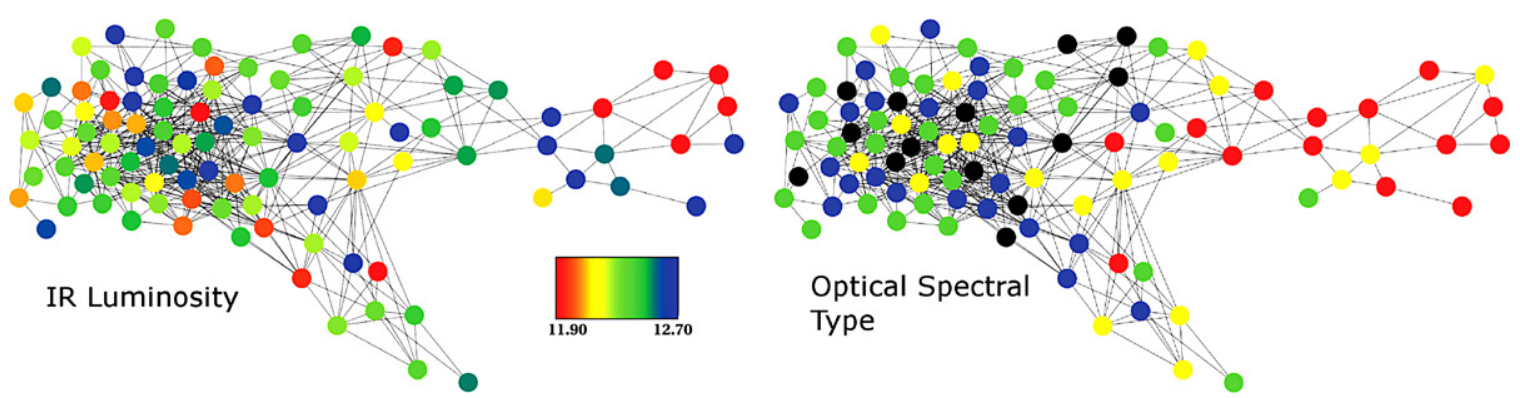

Figure 6. Network diagram, with the nodes color-coded by (left panel) IR luminosity and (right panel) optical spectral class (black: unknown; blue: H II; green: LINER; yellow: Sy2; red: Sy1).

(A color version of this figure is available in the online journal.)
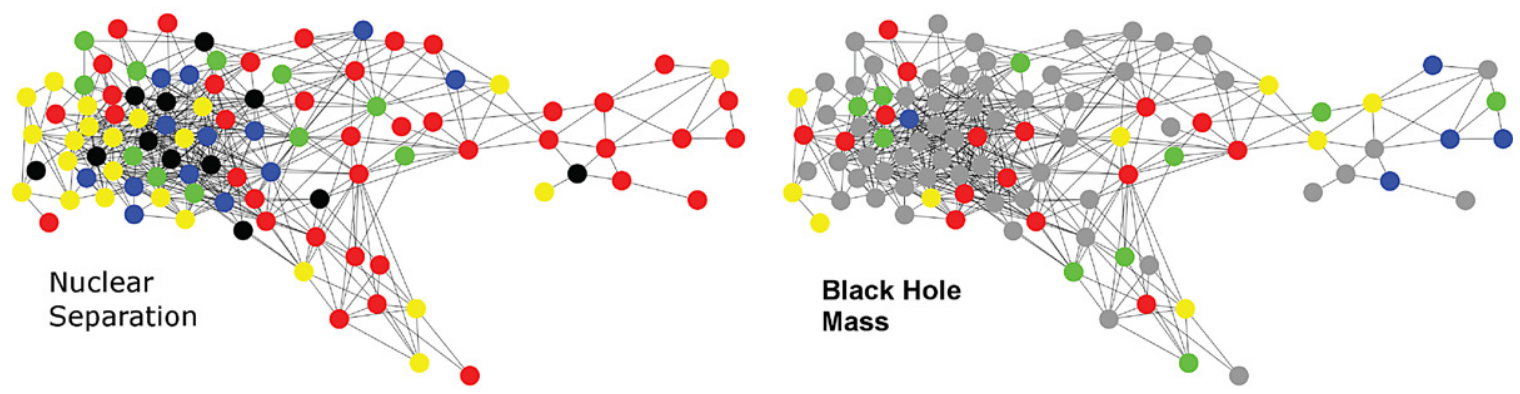

Figure 7. Left panel: network diagram, with the nodes color-coded by projected nuclear separation (black: unknown; blue: $>12 \mathrm{kpc}$; green: 6-12 Kpc; yellow: 0.1-6 Kpc; red: single nucleus). Right panel: black hole mass (black: unknown; blue: $>2.5 \times 10^{8} M_{\odot}$; green: $8.0<M \times 10^{7} M_{\odot}<25.0$; yellow: $5.0<$ $M \times 10^{7} M_{\odot}<8.0 ;$ red: $\left.<5 \times 10^{7} M_{\odot}\right)$.

(A color version of this figure is available in the online journal.)
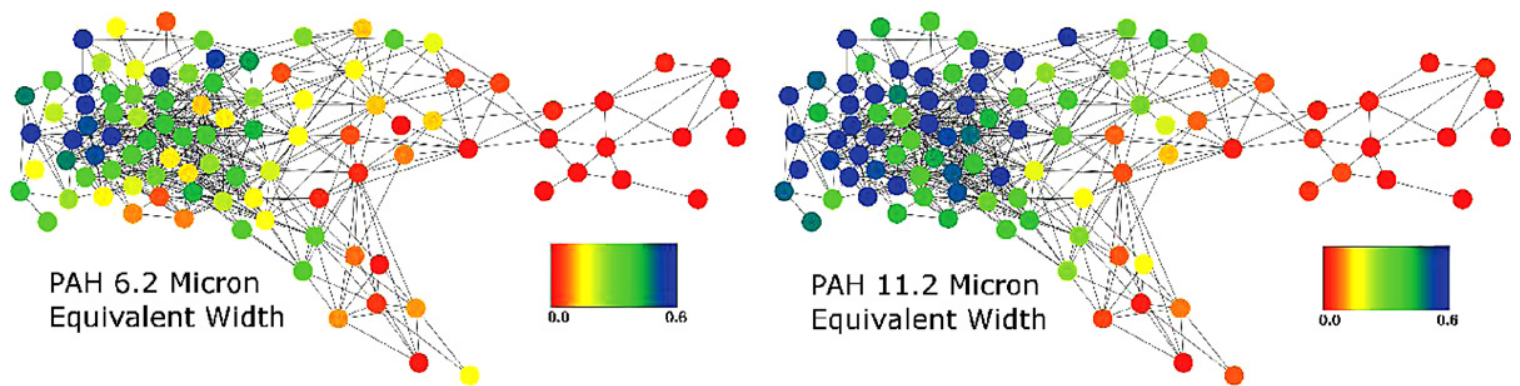

Figure 8. Network diagram, with the nodes color-coded by mid-infrared star formation rate indicators. Left panel: equivalent width of the $6.2 \mu \mathrm{m}$ feature. Right panel: equivalent width of the $11.2 \mu \mathrm{m}$ feature.

(A color version of this figure is available in the online journal.)

grains absorb mid-IR continuum emission from a background source, and in emission when the silicate grains are "hot." It is usually interpreted as a measure of the obscuration toward the central, sub-Kpc nuclear regions. Under this interpretation, a prominent silicate feature is more correlated with AGN activity than star formation-an absorption feature suggests a buried AGN (Imanishi et al. 2007) and an emission feature suggests an unobscured AGN (Hao et al. 2005). There is a caveat in interpreting this network though-the silicate feature is broad, substantially more so than a PAH feature, so its contribution to the $\mathcal{R}$ values will be commensurately larger. ${ }^{16}$ We cannot reliably gauge the magnitude of this effect, so the conclusions that can be drawn from this diagram are limited. We do, however, see trends. The silicate strengths of group A are fairly homogeneous: nearly all are moderately to heavily obscured, with (perhaps) slightly higher values for the outliers. Group C and the first part of group B are more varied, with a wide range

\footnotetext{
${ }^{16}$ As with the IR luminosities, the marginalization over foreground extinction
} should not affect any correlations with silicate strength. of silicate strengths. The nodes at the end of group B universally show negligible absorption, or silicates in emission.

\section{RELIABILITY}

We assess the accuracy and precision, along with possible sources of error, on both the Bayes factors and the network in this section.

\subsection{The Bayes Factors}

We use three methods to examine the behavior of the Bayes factors. First, we assess the precision and accuracy of the whole procedure. We compare what happens to the $\log _{10}(\mathcal{R})$ values in two situations: (1) when the input spectra are intrinsically different and (2) when the input spectra are intrinsically identical but where one has been scaled in luminosity and/or dust extinction. In both situations, we vary the bin-to-bin uncertainties from $2 \%$ to $50 \%$. The results from these simulations are shown in Figure 10. This shows that, as the uncertainties be- 


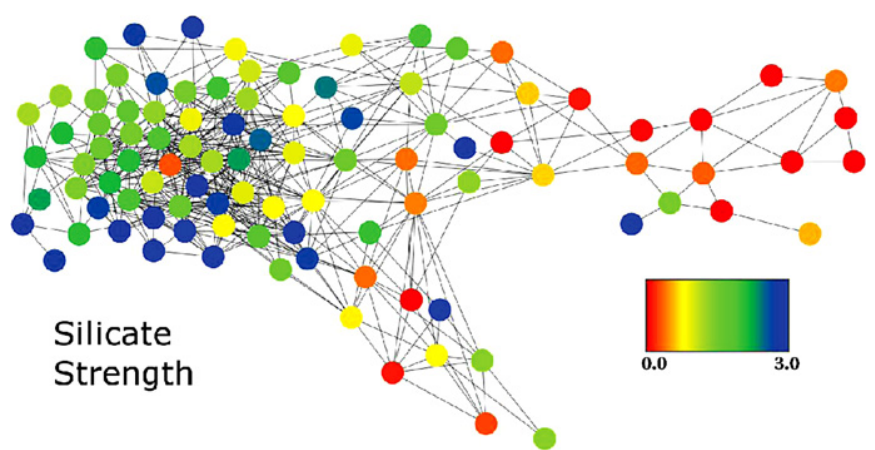

Figure 9. Network diagram, with the nodes color-coded by silicate strength. (A color version of this figure is available in the online journal.)

come smaller, there is a higher probability that $(1) \log _{10}(\mathcal{R})<0$ for those ULIRGs whose intrinsic spectra are the same and (2) $\log _{10}(\mathcal{R})>0$ for those ULIRGs whose intrinsic spectra are different. Hence, the Bayes factors are behaving as expected. When the uncertainties are large there is an almost $100 \%$ overlap of those ULIRGs with "same" and "different" intrinsic spectra. However, the distributions are not centered around zero. We have found that when the uncertainties are increased even further $(\sim 10,000 \%)$, pairings of same and different intrinsic spectra have a mean $\log _{10}(\mathcal{R})$ closer to 1 , indicating that this effect is primarily due to an increasing lack of accuracy in the integration as the uncertainties become smaller and the parameter space becomes more sparse.

Second, we assess the accuracy and precision of the integration algorithm used to compute the Bayes factors. We used the miser algorithm, which is (to our knowledge) the most robust available (Press et al. 1992), but as it is a Monte Carlo algorithm it has uncertainties associated with it which are difficult to compute. ${ }^{17}$ Therefore, we adopt a conservative approach. We compare in Figure 11 the $\log _{10}(\mathcal{R})$ values obtained with the miser algorithm, and an alternative algorithm, VEGAS. The spread is large, with a $1 \sigma$ dispersion on $\log _{10}(\mathcal{R})$ of $\sim 50$. The actual uncertainties due to the limited accuracy/precision of the Monte Carlo integration are, however, much lower than this, as the VEGAS algorithm is less robust than miser. Furthermore, the effect on Figure 4 of uncertainties on individual $\mathcal{R}$ values is likely to be small, as we simply demand that $\log _{10}(\mathcal{R})<0$.

Finally, we examine whether small regions of the spectra dominate the derived $\mathcal{R}$ values. This is important for assessing the reliability of some of the coded variants of Figure 4; the PAH and silicate strength plots repeat (some) information already in the $\mathcal{R}$ values, and so trends may be artificially amplified. We selected 200 pairs at random, and removed from each spectrum a $0.7 \mu \mathrm{m}$ wide region centered on the $11.2 \mu \mathrm{m}$ PAH feature. While there was some variation in the derived $\mathcal{R}$ values, we found that, in $97 \%$ of the cases, $\log _{10}(\mathcal{R})$ did not change sign. We repeated this test for $0.7 \mu \mathrm{m}$ wide regions centered on the $7.7 \mu \mathrm{m}$ PAH feature and in the continuum at $15 \mu \mathrm{m}$, and found similar results. We conclude that, while spectral windows of width $\sim 1 \mu \mathrm{m}$ do contribute to the $\mathcal{R}$ values, they do not dominate them. Therefore, we argue that if a spectral feature spans a small wavelength range, and contains information on a specific physical property, then coding Figure 4 by that feature

\footnotetext{
17 Usually, one can approximate how close one is to the "true" integral through the variance of the integrand. However, in our case, the errors are $\sim 100 \%$ because the parameter space is both sparse and dynamic. Further, it is known a priori that the integrand can never be less than 0 .
}

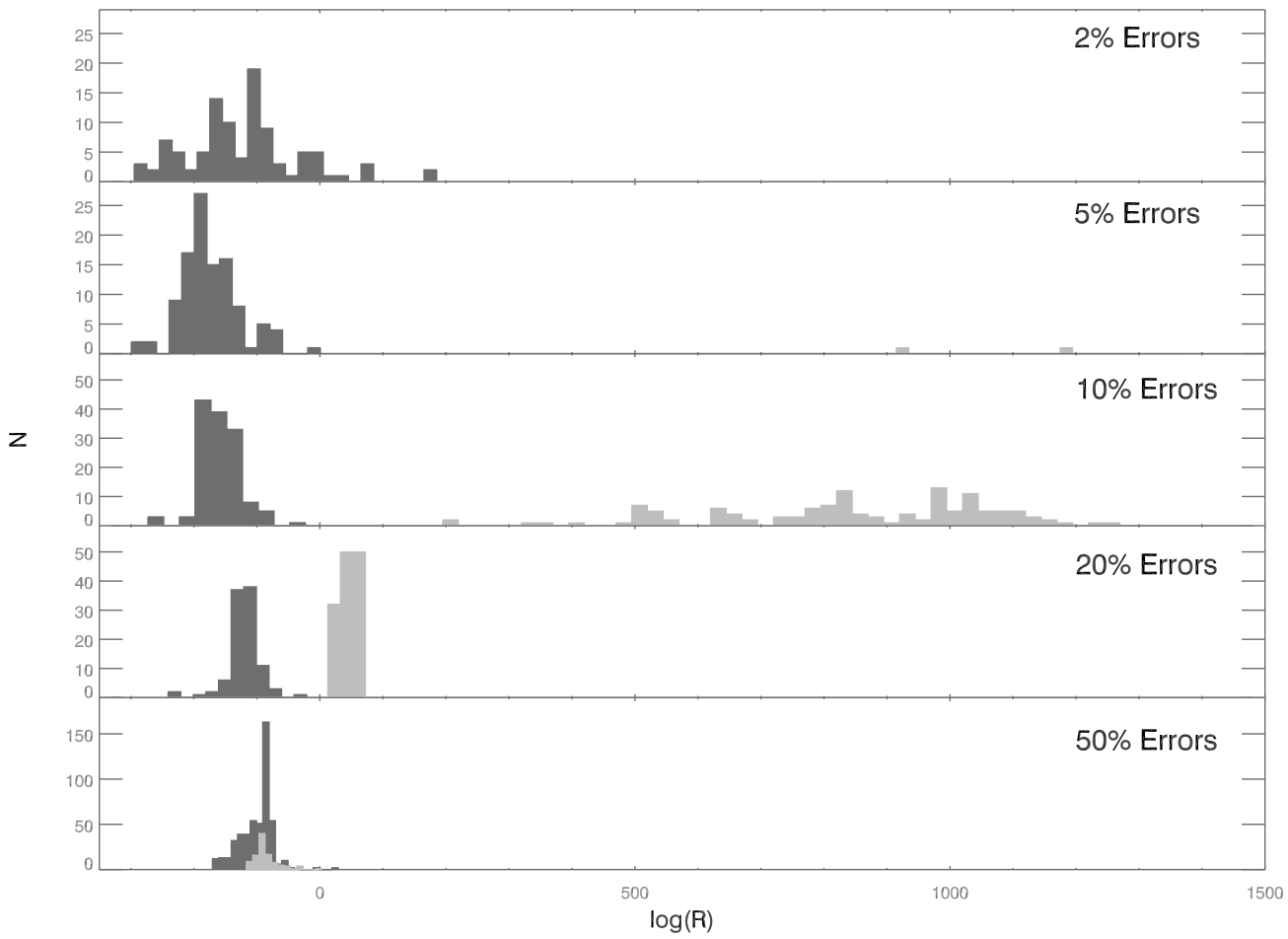

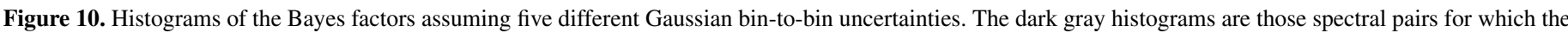

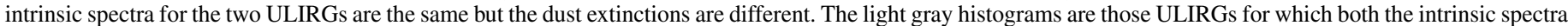

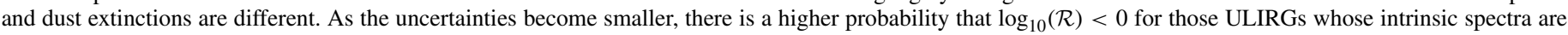

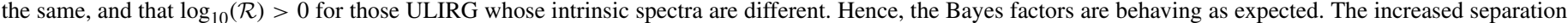

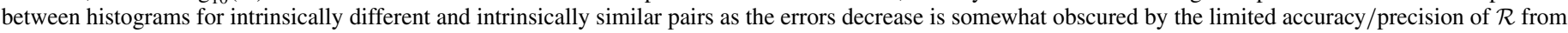
the Monte Carlo integration. 


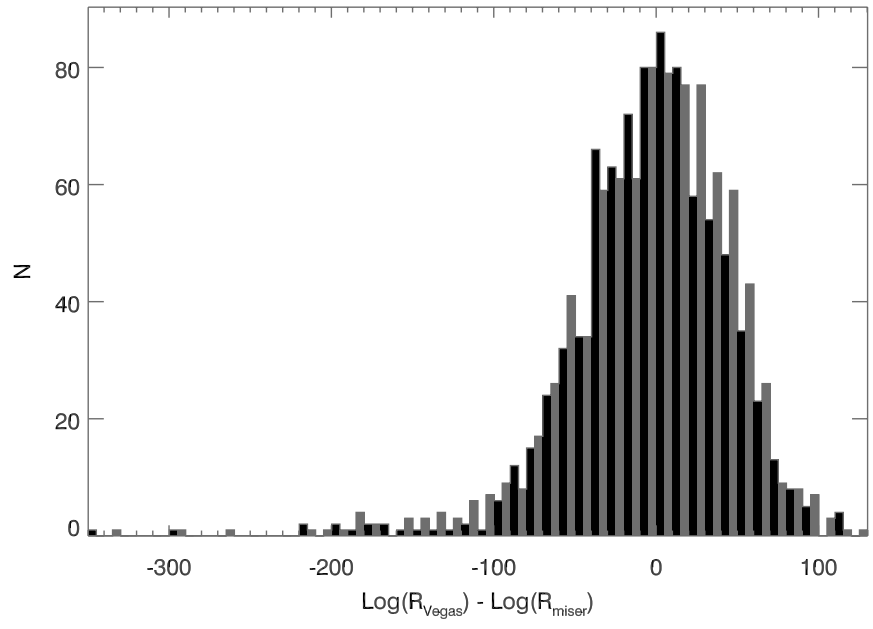

Figure 11. A comparison of the $\log (\mathcal{R})$ values computed using two Monte Carlo algorithms: miser (which we use in our analysis) and VEGAS.

tells us how that property varies with network position, while introducing minimal contamination.

\subsection{The Network}

In this section, we assess four possible sources of error in the methods used to create Figure 4.

First, Figure 4 could simply be randomly connected points, and not contain any "real" structure. We assess this in two ways. Qualitatively, Figure 4 does not resemble a random network with 102 nodes, examples of which are shown in Figure 12. Quantitatively, it has been shown that randomly connected graphs have a Poissonian degree distribution (Erdos \& Renyi 1959). The degree distribution for Figure 4 is shown in Figure 13. It is not well matched to a Poisson distribution.

Second is the effect on Figure 4 from the limited precision of the Bayes factors. The variation in the $\log _{10}(\mathcal{R})$ values due to the Monte Carlo integration is difficult to determine, though we showed in Section 4.1 that the upper limit is $\simeq 50$. So, to assess this, we show in the top panel of Figure 14 the layout obtained after randomly changing all the $\log _{10}(\mathcal{R})$ values by \pm 50 . Even when using the upper limit on the errors, we get essentially the same structure. We conclude that Figure 4 is robust to within the accuracy and precision of the $\mathcal{R}$ values.

Third, one could argue that the structure of Figure 4 is an artifact of the priors, and does not reflect trends in the data. To examine this, we test the sensitivity of Figure 4 to the maximum allowed cold foreground dust extinction, which is the only prior we adopt. Figure 4 assumes a weak upper limit on the cold foreground extinction of $A_{9.7 \mu \mathrm{m}} \simeq 50$ (see Section A.4). In the lower panel of Figure 14, we show the diagram obtained if this limit is relaxed further, to $A_{9.7 \mu \mathrm{m}} \simeq 80$. It closely resembles Figure 4, though there are differences in the number of edges connecting some nodes. As we are using a Bayesian approach, the relaxation of the constraint on $A_{9.7 \mu \mathrm{m}}$ can cause the $\mathcal{R}$ values to rise or fall; for example, IRAS $08572+3915$ is now not connected to any other node and is not plotted. Overall, we conclude that changing the prior on $A_{9.7 \mu \mathrm{m}}$ does not substantially affect the structure of Figure 4.

Fourth, it is possible that the algorithm used to generate the network is a source of error. The methods described in Section 2.2.2 start each network from a random "seed" position, and assume parameters for the attractive and repulsive forces.
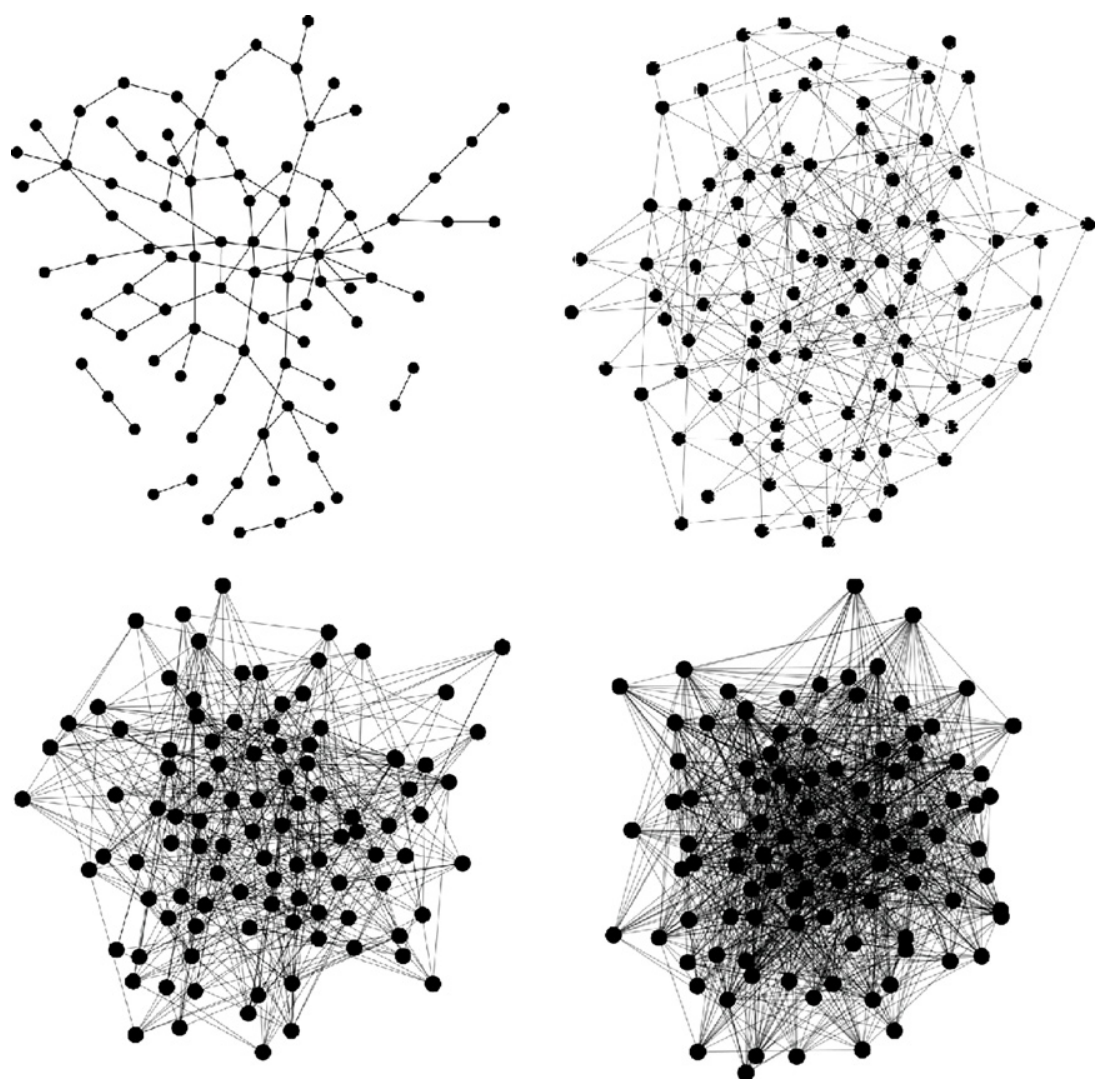

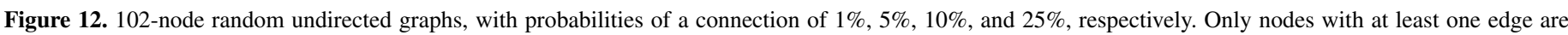
plotted. Qualitatively, these networks do not resemble the network in Figure 4. 


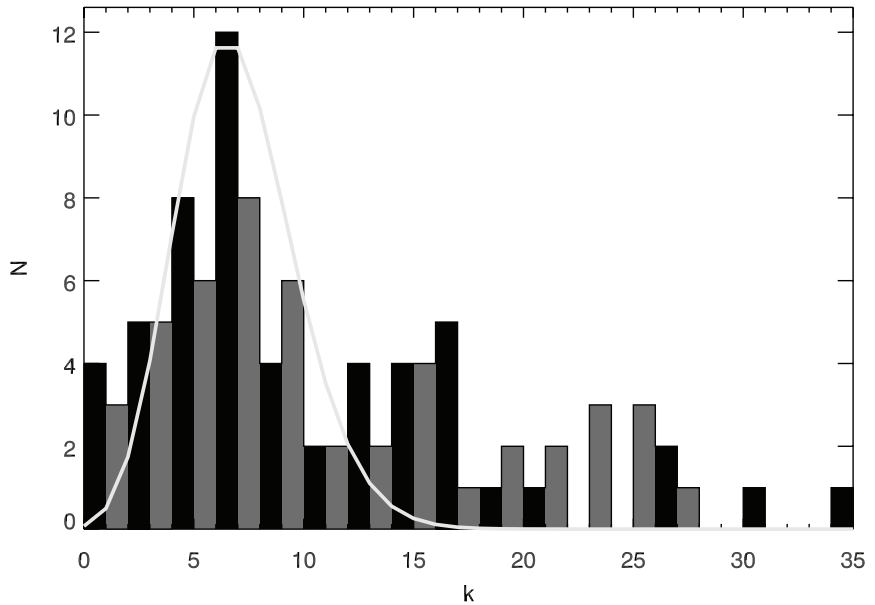

Figure 13. The degree distribution of Figure 4, i.e., the histogram of number of nodes with a given number of connections, $k$. The light gray line is a (scaled) Poisson distribution with a mean of 7 . Our degree distribution is clearly not Poissonian, and so does not follow the degree distribution of a random graph.

Therefore, the final appearance of a network will differ from case to case, and it may be that the seed position or the force parameters are governing the final appearance, not the input data. To test this, we checked to see if different seed positions or different force parameters gave networks without the structure seen in Figure 4 and found they did not. To illustrate, we show in Figure 15 an example of a "raw" network for our data, made using the default parameters for the spring-embedded algorithm within Cytoscape. The same structures can be seen in Figures 4 and 15, the only difference being that the nodes in Figure 15 are too small to identify by number. We conclude that our network is at least reasonably robust to the choice of parameters of the algorithm. This test illustrates a common problem with this type of analysis: the "raw" networks are not always amenable to visual interpretation. This is a particular problem in our case; we want all the nodes to be individually identifiable, but the node labels in the raw networks are invariably too small to see. Therefore, to arrive at Figure 4, we adjusted node/edge properties such that the information in the network was preserved, but in which the individual nodes can be identified.

It is difficult to be certain of the robustness of Figure 4 as this technique has (to our knowledge) not been previously used to examine any astronomical data set, and so there exists no previous study for comparison. We have, however, comprehensively tested the robustness of Figure 4 and not found any significant problems. We conclude that this possibility is remote, and proceed on the assumption that the diagram reflects real trends in the data.

\section{DISCUSSION}

Figure 4 highlights intrinsic similarities in the mid-IR spectra of low-redshift ULIRGs. It places little emphasis on individual spectral features (see Section 4.1), and we have marginalized over IR luminosity, foreground cold dust extinction, and detector noise. Furthermore, as our sample selection is almost unbiased (see Section 2.1), each node is a random snapshot of the ULIRG
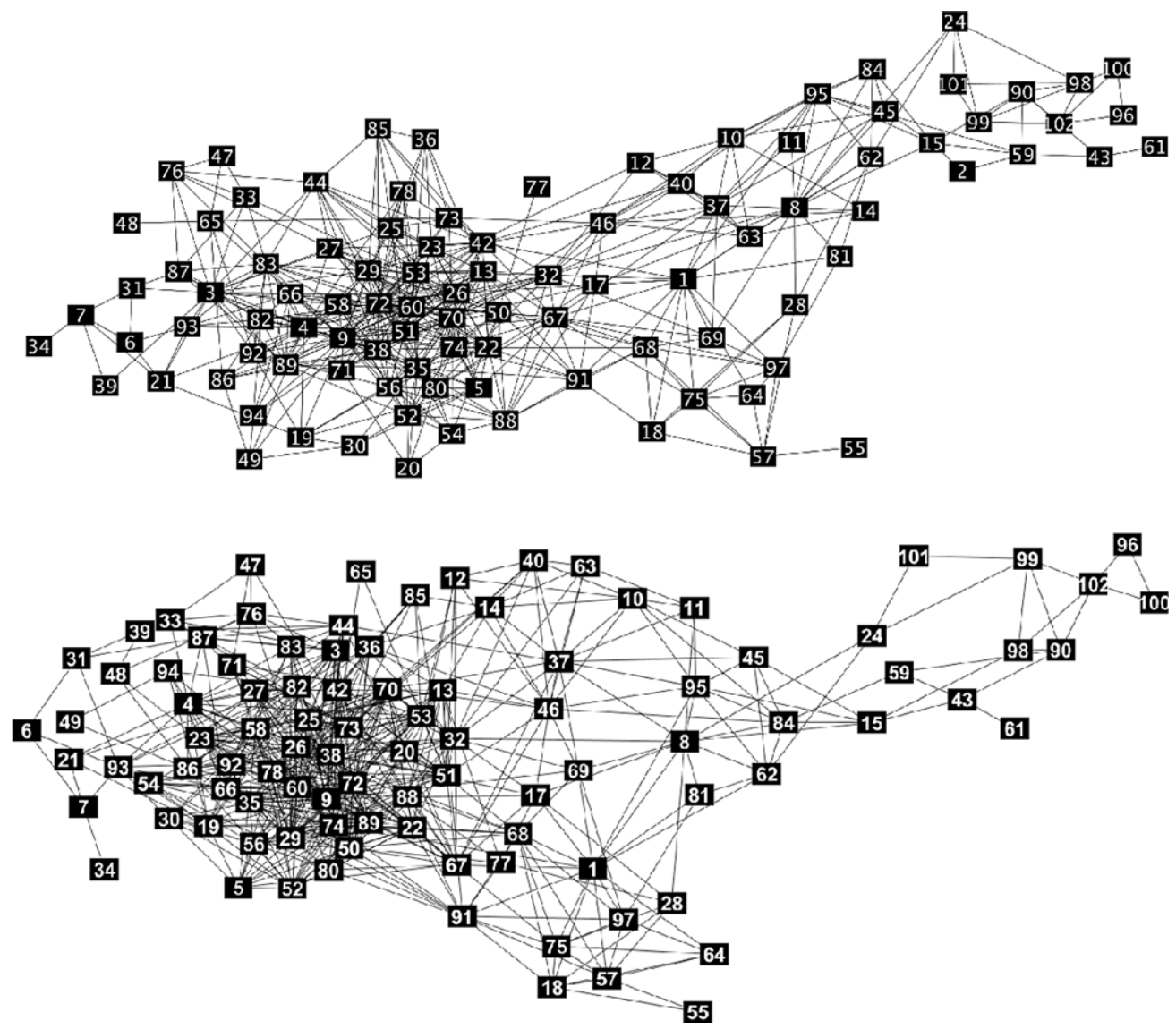

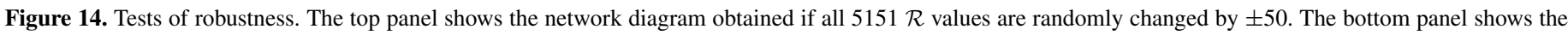
network diagram obtained if the $\mathcal{R}$ values are computed with a relaxed limit on the maximum foreground extinction of $A_{9.7 \mu \mathrm{m}} \simeq 80$, instead of $A_{9.7 \mu \mathrm{m}} \simeq 50$. 


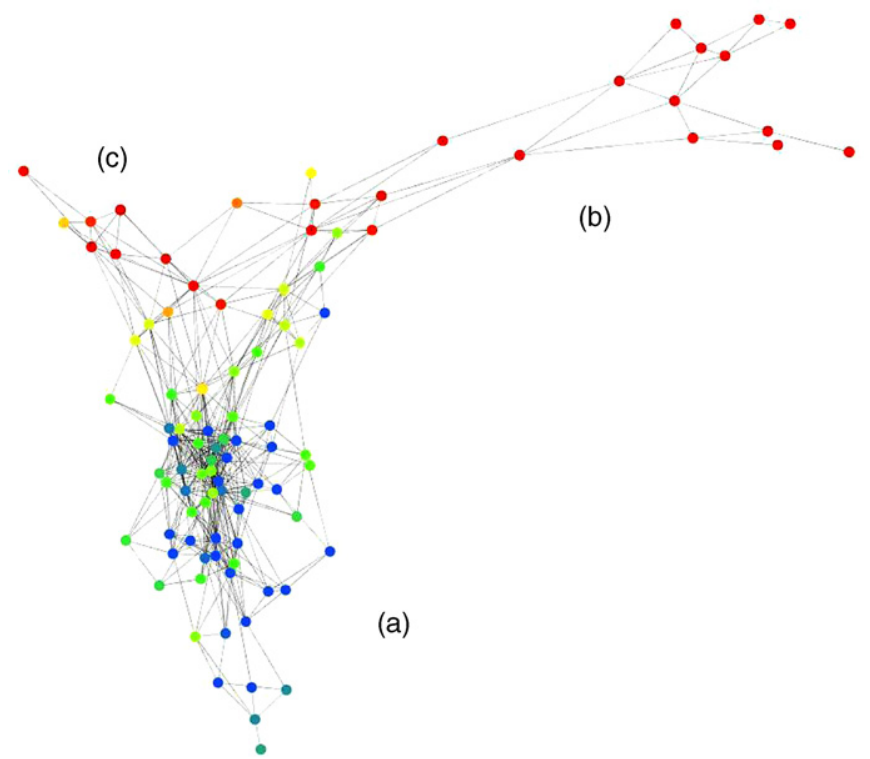

Figure 15. An example of a "raw" network diagram. This figure was made using the same procedures as for Figure 4, but the positions of the nodes have not been subsequently adjusted. The nodes are coded by $11.2 \mu \mathrm{m}$ equivalent width, as in Figure 8.

(A color version of this figure is available in the online journal.)

population. In this section, we explore the conclusions that can be drawn from this diagram and its coded variants. In so doing, we assume that the IRS spectra are a product, on average, of the power sources governing the total IR emission. This assumption is reasonable for the population as a whole, but may break down for individual objects.

\subsection{The Network}

The first conclusion we draw is that, while graph-theorybased approaches are a powerful tool for visualizing complex and heterogeneous data sets, they require a large number of nodes. Our study has 102 nodes, and yet the conclusions we can draw from Figure 4 alone are limited to the subdivision of the sample into two or three groups. Solely from Figure 4, we cannot say if groups A, B, and C are phases in time, or phases in some other variable. Even if we assume they are temporal phases, then it is impossible to say what the time ordering of the groups is. If we increased the number of nodes by an order of magnitude, then the resulting increase in resolution of the network may lead to more insight, but we cannot test this hypothesis here.

Turning to the coded variants of Figure 4 , it is clear that we still lack a complete and homogeneous data set for $z<0.4$ ULIRGs. Several nodes lack morphological and/or optical spectral classifications, and most do not have a dynamical estimate of central BH mass. This omission is serious, given that local ULIRGs are the most easily accessible templates we have for understanding the high-redshift ULIRG population.

We now examine the implications from Figure 4 and its coded variants for the ULIRG population. First, we examine the drivers behind the division of the network into groups $\mathrm{A}, \mathrm{B}$, and $\mathrm{C}$. Three important lines of evidence are (1) the presence of nearly all the widely and moderately separated systems in group A, (2) the fraction of single nucleus systems in groups $\mathrm{B} / \mathrm{C}$ is much higher than in group A, and (3) the generally lower $\mathrm{BH}$ masses in group A compared to group B, though the number of black hole mass measurements is too small for this to have much weight. We therefore propose that groups $\mathrm{A}$ and $\mathrm{B} / \mathrm{C}$ represent temporal phases and are not significantly determined by other factors.

Next, we examine the likelihood of group $\mathrm{C}$ being a separate entity to groups A and B. Group C is unlikely to be outliers to group $\mathrm{A}$ as its nuclear separations and $\mathrm{PAH}$ strengths are different from the outliers on the left-hand side of group A. Instead, it is similar to the first "half" of group B. Based on the lack of connections between groups $\mathrm{B}$ and $\mathrm{C}$, we tentatively propose that group $\mathrm{C}$ is a distinct stage from groups $\mathrm{A}$ and $\mathrm{B}$, but stress that this is not robust. For example, it is conceivable that a source could move from group A to group C and then "jump" to group $\mathrm{B}$, although the lack of a bridge node connecting $\mathrm{C}$ to B means such a jump phase is likely short; as we have $\sim 100$ nodes, the lack of a bridge node suggests a jump phase length of order $2 \%$ or less of the total ULIRG lifetime. ${ }^{18}$

Overall, we propose that groups $\mathrm{A}, \mathrm{B}$, and $\mathrm{C}$ are distinct but overlapping evolutionary phases, with A occurring first, followed by $\mathrm{B}$ and/or $\mathrm{C}$. If a merger remains a ULIRG for most of the duration of the merger, then we can also estimate timescales based on the number of objects in each group; phase A lasts just over half the lifetime of a ULIRG, and phases B and $\mathrm{C}$ last approximately half and one-third the duration of group A, respectively. We do not claim that a ULIRG starts at the left end of A and goes gradually to the right. Instead, we propose that ULIRGs start in group A, with position and intragroup movement determined by unknown factors, and then proceed to $\mathrm{B}$ or $\mathrm{C}$. We also note that the tests of reliability in Section 4.2 involving randomization of the $\mathcal{R}$ values and varying the extinction still gave a clear subdivision of the network into groups $\mathrm{A}$ and $\mathrm{B} / \mathrm{C}$, but groups $\mathrm{B}$ and $\mathrm{C}$ were somewhat "blended" and less distinct. We conclude that the division of the network into " $\mathrm{A}$ versus $\mathrm{B} / \mathrm{C}$ " is more robust than the division "A versus B versus C."

If the network structure is governed by temporal evolution, we can use the purely network based metric of betweenness centrality to make testable predictions. The betweenness centrality (which we term $\mathcal{B}$ ) of a node is the number of shortest paths between other pairs of nodes that pass through that node (Freeman 1979; Brandes 2001). A low $\mathcal{B}$ means the node is inconsequential, while a high $\mathcal{B}$ means the node is an important junction. A suitable analogy would be airports: a regional airport would have a low $\mathcal{B}$, while an international hub would have a high $\mathcal{B}$. It is therefore plausible that a node in the network with a high $\mathcal{B}$ is an archetype of a "transitional" phase that many ULIRGs pass through. Most of the nodes in Figure 4 have $\mathcal{B}$ values in the range $100<\mathcal{B}<400$. Fifteen nodes have $\mathcal{B}$ values in the range $400<\mathcal{B}<800$, while the four unconnected nodes have $\mathcal{B}=0$. Six nodes, however, have what appear to be unusually high $\mathcal{B}$ values: Mrk $231(\mathcal{B}=1850)$, IRAS 00275-2859 (1620), IRAS 03538-6432 (1420), IRAS 05189-2524 (1260), and IRAS 14348-1447 and Mrk 273 (both 1000). We propose that these six objects are examples of key evolutionary phases. Based on their positions in Figure 4, we speculate that Mrk 273 and IRAS 14348-1447 are templates of group A objects, IRAS 03538-6432 is a template of an object transitioning from phase A to phase B, IRAS 05189-2524 is a classic example of an object on the boundary between groups B and C, and Mrk 231 and IRAS 00275-2859 are prime early-B- to late-B-type objects.

\footnotetext{
18 If we assume a total ULIRG lifetime of $10^{8}$ years then the jump phase would be $\lesssim 2 \times 10^{6}$ years long. This is short but feasible; for example, some Wolf-Rayet stars are expected to live approximately this long.
} 


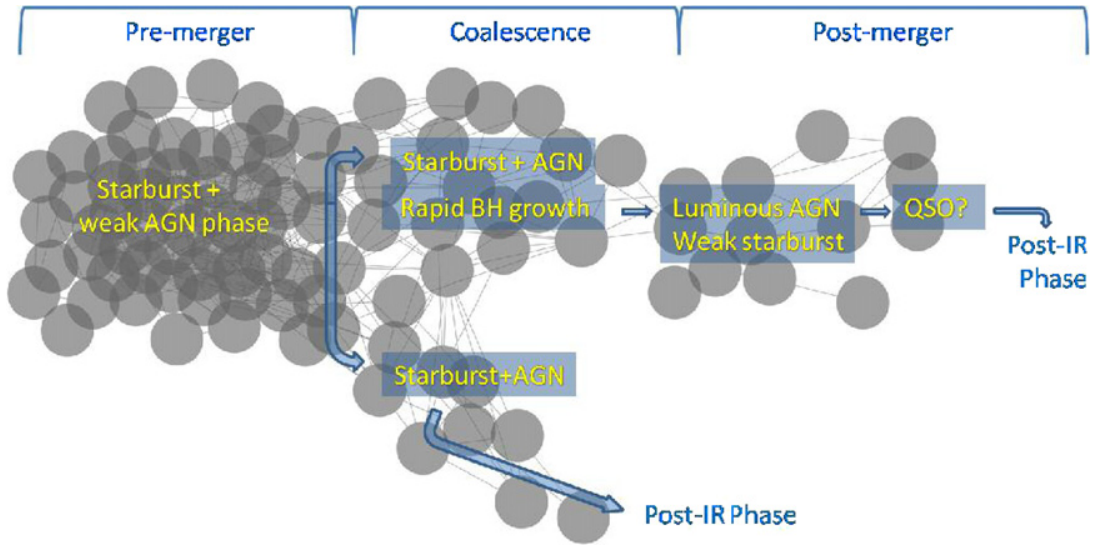

Figure 16. Schematic of the evolutionary scheme described in Section 5.2.

(A color version of this figure is available in the online journal.)

\subsection{The Groups}

We now turn to the properties of groups $\mathrm{A}, \mathrm{B}$, and $\mathrm{C}$. The duration of group $\mathrm{A}$ is hard to quantify, but a reasonable estimate would be from the start of the merger to around the time the progenitor galaxies physically coalesce. This is based on the broad range of projected nuclear separations in this group, from widely separated to single nucleus. The almost universally prominent PAH features suggest that the IR emission is powered mainly by star formation, though this does not preclude the presence of a luminous AGN.

Group A is also highly interconnected, and, as described in Section 3.1, is assortative. In other fields where assortative networks are seen, neighboring nodes tend to identify as common members of a group, and/or have similar properties (see Lusseau \& Newman (2004) for an interesting example). We therefore propose that starbursts in group A are similar, at least to the extent to not give rise to significant differences in the IRS spectra. We propose that outliers to this group are instead caused by heavy intrinsic obscuration (see, for example, Section 5.4), and speculate that there are no large variations in stellar IMF or metallicity from ULIRG to ULIRG.

Phase B follows and overlaps with phase A. Based on the fact that nearly all the objects on the right-hand side of group B have single nuclei, group B likely ends some time after the nuclei of the progenitors have coalesced. We see three interesting trends as we move from left to right in this group. First, the PAHs decline in prominence, becoming negligible (with respect to the continuum) by the right-hand side. Second, silicate absorption varies from strong to weak on the left side, but is universally weak or in emission on the right side. Third, the optical spectral types are varied in the first half but almost universally Seyferts in the second half. We therefore propose that the relative contribution from star formation to the midIR emission declines as we move from left to right, while the contribution from an AGN increases, ${ }^{19}$ until some objects at the end of this phase briefly become optical QSOs. This is consistent with previous studies which show that the AGN fraction increases with increasing merger age (e.g., Veilleux et al. 2002, 2006, though see also Rigopoulou et al. 1999), and suggests either that the accretion rate has increased upon moving into group B, and/or that the central $\mathrm{BH}$ has reached a "threshold" mass for luminous AGN activity. We further propose

\footnotetext{
19 In contrast to phase A, where we make no claims relating a ULIRG's
} intragroup position to its evolutionary stage. that the heterogeneity of phase B arises from two factors. First is varying amounts of gas/dust driven into the nuclear regions. Second is AGN feedback; a luminous AGN can generate nuclear or galactic-scale winds, and the effects of these winds will vary substantially from case to case.

Two examples lend support to our proposal that AGNs become more luminous and less obscured as we move through phase B. First, IRAS 19254-7245 (object 81, also known as the Superantena) is located where we expect the AGN to be intrinsically luminous but still deeply buried, an expectation that appears to be borne out by recent Suzaku observations (Braito et al. 2009). Second, Mrk 231 (object 8) is located where an AGN-driven wind may be expected, and indeed this object is thought to contain a starburst, an energetic AGN, and a nuclear outflow (Lípari et al. 2005).

Assuming that phase $\mathrm{C}$ is a separate evolutionary stage, then it is difficult to interpret as it contains a small number of objects. It seems to have similar properties to the first half of phase B, except perhaps for the nuclear separations, which may be smaller, on average, in phase $\mathrm{C}$. Therefore, we propose that this phase also follows phase A, and that it is characterized mainly by waning star formation. We do not see evidence for a substantial increase in AGN activity in this group, and so propose that this phase is shorter in time than group B, and that its members are unlikely to become optical QSOs.

We show a cartoon-type diagram of this scheme in Figure 16.

If all ULIRGs start off in group A, then the obvious question is what determines if they go to phase B or phase C $?^{20}$ Broadly, there are two possible drivers; the dynamics/morphology of gas and dust (e.g., how much is available, and how efficiently it is channeled into the nuclear regions), or seed $\mathrm{BH}$ mass (models suggest the minimum seed BH mass for AGN activity in ULIRGs is $\sim 10^{7} M_{\odot}$ (Taniguchi et al. 1999)). If the latter is the driver, and the end product of a ULIRG is an elliptical galaxy (e.g., Genzel et al. 2001; Dasyra et al. 2006b) then the antecedents of phase $\mathrm{C}$ should have smaller mass bulges than phase B. As there is no plausible evidence for a bimodality in $\mathrm{BH} /$ bulge masses in ellipticals, we think it more likely that the end products of $\mathrm{B}$ and $\mathrm{C}$ are similar, except that some aspect of the merger dynamics of objects in B allows some of them to go through a brief optical QSO phase.

This evolutionary picture fits well with recent studies of IRluminous galaxies. The interconnected nature of Figure 4 im-

\footnotetext{
20 Assuming they cannot do both, but see Section 5.1.
} 


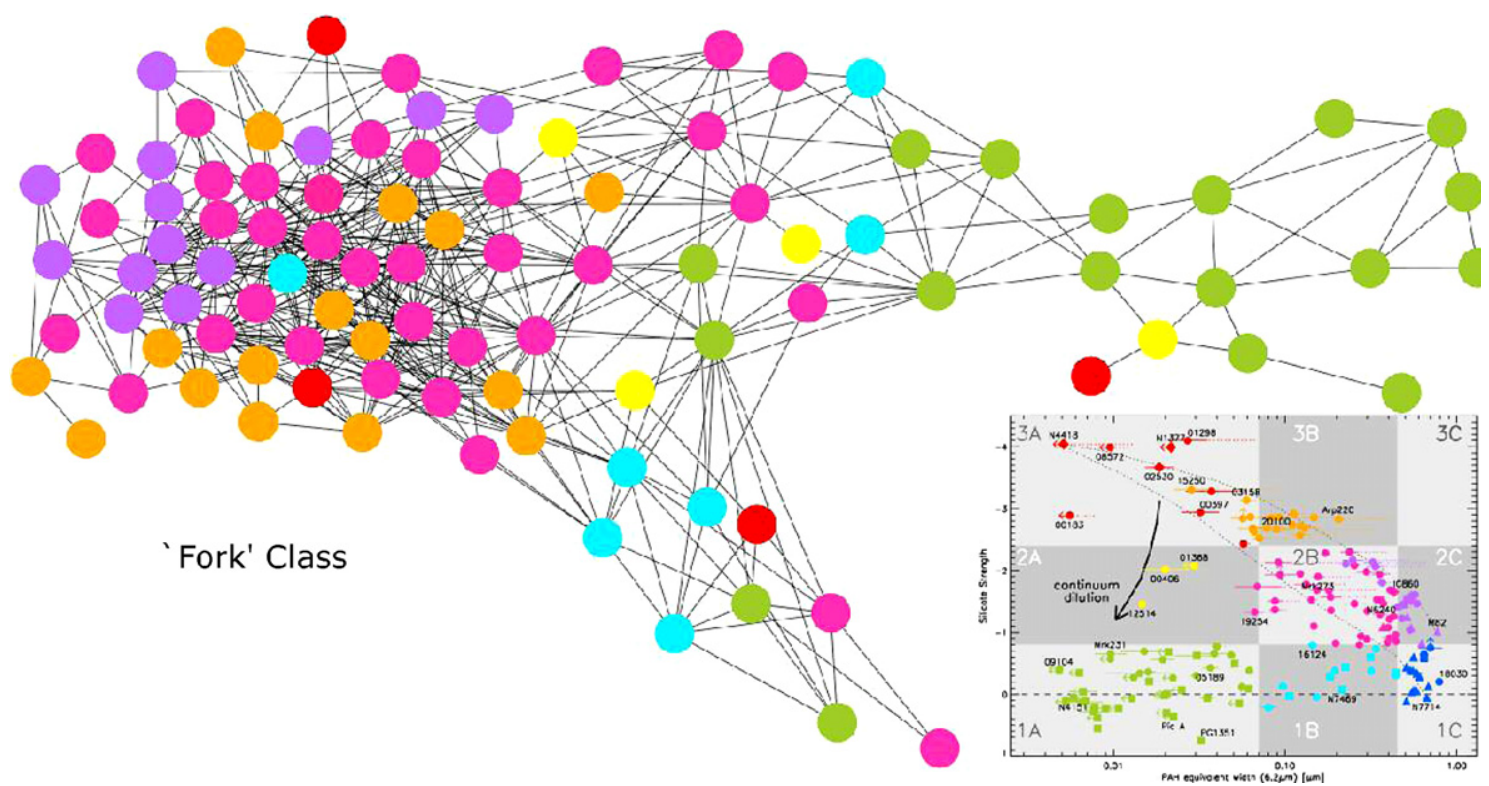

Figure 17. Network diagram, with the nodes color-coded by their "Fork" classification (Spoon et al. 2007). A copy of the Fork diagram has been embedded for reference.

(A color version of this figure is available in the online journal.)

plies that the starburst and AGN activity arises from a common physical mechanism, which tallies with imaging studies of ULIRGs, which show them all to be mergers (Surace et al. 2000; Cui et al. 2001; Farrah et al. 2001; Bushouse et al. 2002; Veilleux et al. 2002, 2006). The location of all the QSOs in the sample at the end of group $\mathrm{B}$, and their low $\mathcal{B}$ values, suggests that few ULIRGs pass through a phase where they are simultaneously ULIRGs and quasars, and/or that the ULIRG-QSO phase is brief, in agreement with recent work (Farrah et al. 2001; Tacconi et al. 2002; Kawakatu et al. 2006, 2007). Our picture takes the idea that IR-luminous starbursts are present in most ULIRGs, while IR-luminous AGNs are present in just under half (Genzel et al. 1998; Rigopoulou et al. 1999; Tran et al. 2001; Klaas et al. 2001; Farrah et al. 2003; Franceschini et al. 2003; Vega et al. 2008) and extends it by providing (1) a single diagrammatic representation of the ULIRG evolutionary plane, (2) groupings into evolutionary phases, and (3) descriptions of the properties of these phases, including homogeneity, timescales, and power source.

Finally, we note a peculiar aspect of the diagrams in Figure 8: the remarkable homogeneity of the $11.2 \mu \mathrm{m}$ PAH strengths in group A, and the smoother gradient of $11.2 \mu \mathrm{m} \mathrm{PAH} \mathrm{strengths}$ through groups $\mathrm{B}$ and $\mathrm{C}$, in comparison to the $6.2 \mu \mathrm{m} \mathrm{PAH}$ strengths. We do not have a plausible explanation for this. It could, for example, be highlighting an important part of the way in which PAHs diagnose star formation rates, or a subtle systematic error in our calculations. We do not consider this point further here, but highlight it as an interesting avenue to pursue in future work.

\subsection{Comparison to Other Mid-IR Classification Schemes}

As our evolutionary framework is based mostly on mid-IR spectroscopy, it is interesting to compare it to previous work in this field. A recent example is that of Spoon et al. (2007) who published a mid-infrared based classification scheme (the "Fork" diagram, their Figure 1) for IR-luminous galaxies. The "Fork" diagram and our network diagram overlap significantly in information use, as the Fork diagram uses both the silicate feature and the $6.2 \mu \mathrm{m}$ PAH feature, but our diagram includes every other part of the spectrum, and is constructed using a fundamentally different methodology. Our intention, though, is to compare the predictions from the two schemes, not to perform an independent check.

In Figure 17, we code each node by its classification in the Fork diagram. We see a clear delineation. With a single exception, all the objects in group A reside in the upper branch of the Fork diagram (classes $2 A / 2 B / 2 C$; hereafter, Fork classes are given in italics). Group $B$ on the other hand contains all the class $2 A$ objects, nearly all the class $1 A$ objects, and a few $1 B /$ $2 B / 3 A$ objects. Finally, group $C$ contains the remaining class $2 A$ 's, some $1 B$ 's, and a few $2 B$ 's and $3 A$ 's.

This indicates that the two schemes are crudely in agreement. Spoon et al. (2007) suggest an evolutionary picture in which sources move up the diagonal branch in their Figure 1 ( $2 C \rightarrow$ $2 B \rightarrow 3 B \rightarrow 3 A$ ), before either dropping vertically downward $(3 A \rightarrow 1 A)$ or via the slanted branch back to $I C$ and on from there to $1 A / 1 B$. In Figure 9, we see a trend where the $2 C / 2 B / 3 B$ sources lie on the left-hand side, with the $1 A$ sources lying on the right-hand side. Figure 17, however, provides a more nuanced diagnostic. From it, we can discern two distinct evolutionary paths, and that the $1 B$ and $2 A$ classes are likely starburst/AGN transition classes, rather than just the $2 A$ class.

\subsection{Notable Objects}

The positions of some famous objects are interesting. We describe some of them in this section.

Arp 220 (object 7) is frequently used as a template for highredshift objects. Its presence in group A marks it as young. It is connected by only four edges, making it an atypical example of a local ULIRG. This does not preclude its use as a template, but suggests it is not suitable to use if one is interested in determining if high-redshift ULIRGs resemble local ones. From Section 5.1, its outlier status arises due to heavy intrinsic obscuration, which agrees with the properties of its mid-IR spectra; it shows strong PAHs, but also strong silicate absorption, and a steeply rising continuum at $>10 \mu \mathrm{m}$.

Next, we consider IRAS F00183-7111 (object 11). This object is atypical (only two edges), and harbors an obscured 
AGN that is close to burning through its dust cocoon. An independent study comes to similar conclusions; Spoon et al. (2009), using the high-resolution IRS spectra to look for outflow signatures in fine structure lines (i.e., information that is not contained in the low-resolution spectra) show that this object contains an obscured nuclear outflow driven by an AGN.

Finally, the four objects in Table 1 that are not plotted in Figure $4(16,41,48,79$; one could also include object 2 here as its single connection depends on the assumptions made when computing $\mathcal{R}$ ) are interesting because their lack of connection is unusual. We defer a complete discussion on these sources to a future paper, and here only briefly describe some possibilities. Part of the reason for this may be luminosity dependent; object 16 (IRAS 00397-1312) is the brightest in the sample, albeit by a small margin, and object 41 (IRAS 07145-2914) is the faintest by over an order of magnitude. It may also be due to a combination of unusual spectral properties; object 16 (IRAS 00397-1312) has the deepest CO absorption feature of any object in the sample, and object 79 (IRAS 18030+0705) has extraordinarily strong $\mathrm{PAH}$ features. It is possible therefore that these objects represent either a very brief and/or very rare evolutionary stage, or that the merger dynamics are highly atypical in some way.

\section{CONCLUSIONS}

We have taken a large mid-infrared spectroscopic database of low-redshift ULIRGs, and applied to it two novel analysis methods: (1) a Bayesian based estimator of similarities between pairs of spectra that takes into account every spectral resolution element, marginalizing over luminosity, foreground obscuration, and instrument noise, and (2) a visualization algorithm based on force-directed networks that efficiently presents these similarities across the sample simultaneously. We combine these results with archival data to propose, with some reserve, the following evolutionary description for ULIRGs in the low-redshift universe:

1. The IR emission in ULIRGs is consistent with being driven by a single underlying physical process. We see no evidence for multiple, separate evolutionary tracks. There is, however, evidence for at least two and possibly three evolutionary subphases.

2. The first phase (phase A), through which all ULIRGs go, lasts from the initial encounter until approximately coalescence. The IR emission arises mainly from star formation, with a contribution from an AGN in some cases. The highly interconnected, assortative nature of phase A suggests that there is little variation in starburst parameters from object to object, with observed variations in the IRS spectra instead caused largely by differing foreground obscuration.

3. At around the time the progenitors start to coalesce, a ULIRG can branch off into one of two phases. We suggest that the track a ULIRG takes depends primarily on the initial impact parameters and dynamics of the merger and the availability of gas/dust, and to a lesser extent on the masses of the central BHs in the merger progenitors.

4. The first of these two phases (phase B) lasts approximately half the length of phase $\mathrm{A}$. The relative contribution from star formation to the mid-IR emission declines as we move from left to right, while the contribution from an AGN increases, until some objects near the end of this phase briefly become optical QSOs. Phase B is less interconnected and more heterogeneous than phase $\mathrm{A}$, implying that more than just foreground obscuration is driving the shape of the IRS spectra. We propose that this increased heterogeneity arises from two factors: (1) varying amounts of gas/ dust driven into the nuclear regions by differing merger dynamics, and (2) feedback effects from AGN-driven winds.

5. The second phase (C) lasts about one-third the length of phase A. It is similar to phase B in that the mid-IR spectra are heterogeneous, but the decline in luminosity of the starburst relative to the AGN is less pronounced. Few or no systems on this track pass through a QSO phase.

6. We use the graph-theory-based metric of node betweenness centrality to identify six ULIRGs that may be archetypes of key points in this evolutionary cycle. We propose that Mrk 273 and IRAS 14348-1447 are examples of phase A objects, IRAS $03538-6432$ is a prime example of an object transitioning from phase A to phase B, IRAS 05189-2524 is an example of an object on the boundary between phase B and phase C, and Mrk 231 and IRAS 00275-2859 are prime early-B- to late-B-type objects.

7. The $11.2 \mu \mathrm{m}$ PAH feature appears to be a remarkably good diagnostic of the evolutionary phase of a ULIRG, more so than the $6.2 \mu \mathrm{m}$ PAH feature or the $9.7 \mu \mathrm{m}$ silicate absorption feature. Even though we are using the entire spectral range to construct the network, all the objects in group A have prominent, homogeneous $11.2 \mu \mathrm{m}$ equivalent widths, which then smoothly decline as we move left to right through groups $\mathrm{B}$ and $\mathrm{C}$, until we reach the right-hand side of both groups where the PAHs are negligible.

We thank the referees for very helpful reports that greatly improved the clarity of the paper, and Steve Young at Hamilton College for computer technical support. This work is based on observations made with the Spitzer Space Telescope, and has made extensive use of the NASA/IPAC Extragalactic Database, both of which are operated by the Jet Propulsion Laboratory, California Institute of Technology under contracts with NASA. This project was supported in part by NSF grant CHE-0116435 as part of the MERCURY supercomputer consortium. ${ }^{21}$ Support for the IRS GTO team at Cornell University was provided by NASA through Contract Number 1257184 issued by JPL/Caltech. D.F. and S.O. thank the Science and Technologies Facilities Council for support. N.C. gratefully acknowledges support from a Cottrell College Science Award from the Research Corporation. Funding for Cytoscape is provided by a federal grant from the U.S. National Institute of General Medical Sciences (NIGMS) of the National Institutes of Health (NIH) under award number GM070743-01 and the U.S. National Science Foundation (NSF). Corporate funding is provided through a contract from Unilever PLC. Network Workbench was supported in part by the NSF IIS-0513650 award.

\section{APPENDIX}

\section{MATHEMATICAL DETAILS}

\section{A.1. Introduction}

The formalism for determining the degree of similarity between two data vectors using a Bayes factor was first developed

\footnotetext{
21 http://mercury.chem.hamilton.edu.
} 


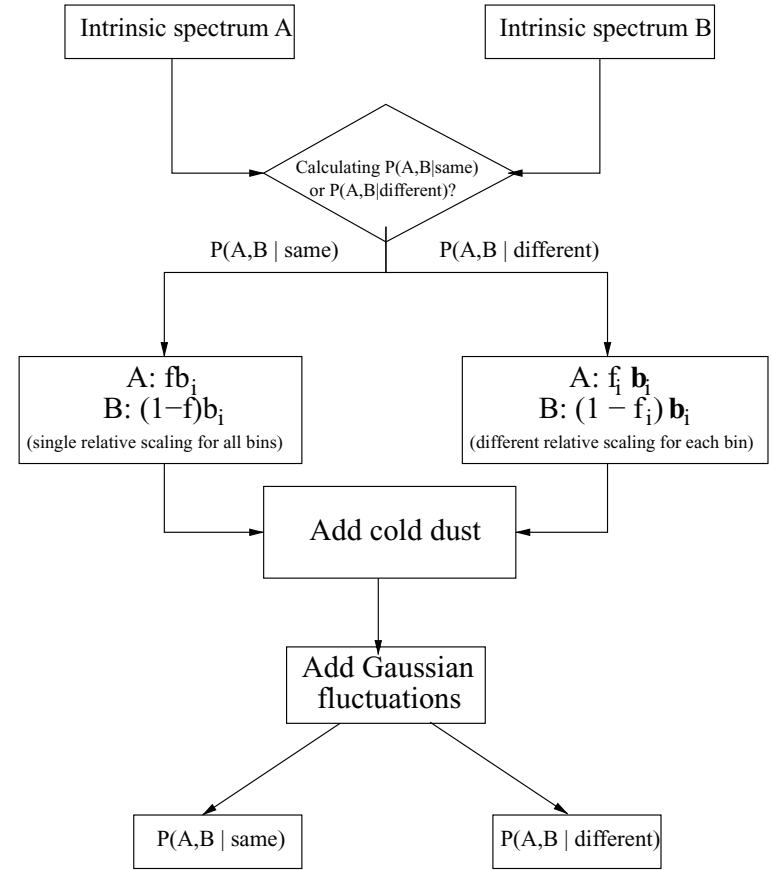

Figure 18. Flowchart of the method used to calculate $\mathcal{R}$. For each pair of spectra, the flowchart is followed twice: once to calculate $P(\mathrm{~A}, \mathrm{~B} \mid$ different $)$ and once to calculate $P(\mathrm{~A}, \mathrm{~B} \mid \mathrm{same})$.

in detail in Jeffreys (1961), who quantifies the belief that two flux measurements using different detectors with different acceptances are measuring the same flux. This, in essence, reduces to whether or not the differences between the two measured fluxes are what is expected if the assumptions about the acceptances of the detectors are correct.

We here extend this formalism in two ways: (1) we extend the calculation to involve not just one measurement per experiment, but many measurements from many wavelength bins (i.e., a spectrum), and (2) we incorporate the ability to marginalize over external variables (e.g., luminosity, dust extinction) so that contributions from those variables to the $\mathcal{R}$ values can be accounted for. The procedure is shown as a flow chart in Figure 18.

\section{A.2. Method}

We start by defining the flux in the $i$ th wavelength bin of spectrum A as $f_{i}^{\prime} b_{i}$, where $b_{i}$ ranges from 0 to $\infty$, and $f_{i}^{\prime}$ from 0 to 1 . If the fluxes are given in photon counts, then $b_{i}$ is the mean number of photons in the $i$ th bin of both spectra, $f_{i}^{\prime}$ is the probability that the photons are emitted from source A, and $1-f_{i}^{\prime}$ is the probability that the photons are emitted from source B. The number of photons in the $i$ th bin of spectrum B is therefore $\left(1-f_{i}^{\prime}\right) b_{i}$. An analogy would be collecting balls into two receptor bins with different probabilities of accepting an individual ball; in this case, $b_{i}$ is the mean number of balls that will enter both bins, $f_{i}^{\prime}$ is the relative acceptance of one bin, and $\left(1-f_{i}^{\prime}\right)$ is the relative acceptance of the other.

If the two spectra are identical, then we will see equal contributions from the two spectra to each bin

$$
f_{i}^{\prime} b_{i}=\left(1-f_{i}^{\prime}\right) b_{i},
$$

and therefore it follows that

$$
f_{i}^{\prime}=f=\frac{1}{2},
$$

where $f$ and $(1-f)$ are the "true" fluxes emitted by sources A and $\mathrm{B}$, respectively. In other words, to ask whether or not the sources have the same intrinsic spectrum is equivalent to asking whether or not $f_{i}^{\prime}=f$.

Now, if the two spectra differ, but only by a multiplicative scaling factor, and if $N_{A}$ and $N_{B}$ are the normalizations for spectra $\mathrm{A}$ and $\mathrm{B}$, respectively, then

$$
f_{i}^{\prime}=f=\frac{N_{A}}{N_{A}+N_{B}} .
$$

Note that $f$ would be the same for all the bins if the two sources were the same (that is, if they differed only by a normalization factor). We will not assume a specific value of $f$ a priori; hence it must be marginalized (i.e., integrated out, thereby accounting for all possibilities for $f$ ). It is important to note that if the sources are intrinsically different, then $f_{i}^{\prime}$ is not constrained by $f$.

It is now straightforward to expand Equation (1) in terms of $f, f_{i}^{\prime}$, and $b_{i}$ :

$$
\begin{aligned}
\mathcal{R} & =\frac{P(\mathrm{~A}, \mathrm{~B} \mid \text { different })}{P(\mathrm{~A}, \mathrm{~B} \mid \text { same })} \\
& =\frac{\int_{0}^{1} d \vec{f}^{\prime} \int_{0}^{\infty} d \vec{b} P\left(\mathrm{~A}, \mathrm{~B} \mid \vec{f}^{\prime}, \vec{b}\right) P\left(\vec{f}^{\prime}, \vec{b} \mid \text { different }\right)}{\int_{0}^{1} d f \int_{0}^{\infty} d \vec{b} P(\mathrm{~A}, \mathrm{~B} \mid f, \vec{b}) P(f, \vec{b} \mid \text { same })},
\end{aligned}
$$

where $P(f, \vec{b} \mid$ same $)$ and $P\left(\vec{f}^{\prime}, \vec{b} \mid\right.$ different $)$ are the prior densities, which encode any information that might be known about $\vec{b}, \vec{f}^{\prime}$, and $f$ before the data are taken. For a Bayes factor to be well defined, the priors that enter the calculation must be proper probability densities; that is, they must integrate to one. However, as noted below, for this problem we are able to finesse this restriction.

Let us consider each of the terms in Equation (A4) in turn. If the counts in the $i$ th bins of the two spectra follow Poisson statistics, then

$$
\begin{aligned}
P\left(\mathrm{~A}, \mathrm{~B} \mid \vec{f}^{\prime}, \vec{b}\right)= & \prod_{i=1}^{D} \frac{\left(f_{i}^{\prime} b_{i}\right)^{N_{i}} e^{-f_{i}^{\prime} b_{i}}}{N_{i} !} \\
& \times \frac{\left(\left(1-f_{i}^{\prime}\right) b_{i}\right)^{M_{i}} e^{-\left(1-f_{i}^{\prime}\right) b_{i}}}{M_{i} !},
\end{aligned}
$$

where $D$ is the number of wavelength bins in both spectra and $f_{i}^{\prime} b_{i}$ and $\left(1-f_{i}^{\prime}\right) b_{i}$ are interpreted as the mean photon count in the $i$ th bin of spectra A and $\mathrm{B}$, respectively; $N_{i}$ and $M_{i}$ are the numbers of photons that are present in this bin of spectra $\mathrm{A}$ and $\mathrm{B}$, respectively. Note that $f_{i}^{\prime}$ is allowed to vary independently in each bin. If the spectra are different, as is assumed to be the case when we are calculating $P\left(\mathrm{~A}, \mathrm{~B} \mid \vec{f}^{\prime}, \vec{b}\right)$, then there is no constraint on the relative normalizations of the two spectra from bin to bin.

When calculating the probability of obtaining the two spectra given that they are the same, we must consider

$P(\mathrm{~A}, \mathrm{~B} \mid f, \vec{b})=\prod_{i=1}^{D} \frac{\left(f b_{i}\right)^{N_{i}} e^{-f b_{i}}}{N_{i} !} \frac{\left((1-f) b_{i}\right)^{M_{i}} e^{-(1-f) b_{i}}}{M_{i} !}$.

In contrast to Equation (A5), the spectra here are only expected to have different overall normalizations and fluctuate according to Poisson statistics.

We now consider the priors in Equation (A4) where we shall assume flat priors for $f_{i}^{\prime}, f$, and $\vec{b}$, defined initially on compact 
sets. We also assume that $f_{i}^{\prime}$ and $\vec{b}$ are independent a priori, as are $f$ and $\vec{b}$. That is

$$
\begin{aligned}
P(f, \vec{b} \mid \text { same }) & =P(f \mid \vec{b}, \text { same }) P(\vec{b} \mid \text { same }) \\
& =P(f \mid \text { same }) P(\vec{b} \mid \text { same }) .
\end{aligned}
$$

We take the prior for $f$ to be

$$
P(f \mid \text { same })=\frac{1}{f_{\max }-f_{\min }},
$$

where $f_{\min } \rightarrow 0$ and $f_{\max } \rightarrow 1 .{ }^{22}$ Similarly,

$$
P(\vec{b} \mid \text { same })=\frac{1}{\left(b_{\max }-b_{\min }\right)^{D}},
$$

where $b_{\min } \rightarrow 0$ and $b_{\max } \rightarrow \infty$. And so,

$$
P(f, \vec{b} \mid \text { same })=\frac{1}{\left(f_{\max }-f_{\min }\right)\left(b_{\max }-b_{\min }\right)^{D}} .
$$

Similarly,

$$
\begin{gathered}
P\left(\vec{f}^{\prime}, \vec{b} \mid \text { different }\right)=P\left(\vec{f}^{\prime} \mid \vec{b}, \text { different }\right) P(\vec{b} \mid \text { same }) \\
=P\left(\vec{f}^{\prime} \mid \text { different }\right) P(\vec{b} \mid \text { same }),
\end{gathered}
$$

and likewise for $f_{i}^{\prime}$

$$
P\left(\vec{f}^{\prime} \mid \text { different }\right)=\frac{1}{\left(f_{\max }^{\prime}-f_{\min }^{\prime}\right)^{D}},
$$

where $f_{\min }^{\prime} \rightarrow 0$ and $f_{\max }^{\prime} \rightarrow 1$. Finally, we assume

$$
P(\vec{b} \mid \text { different })=\frac{1}{\left(b_{\max }-b_{\min }\right)^{D}} .
$$

Therefore,

$P\left(\vec{f}^{\prime}, \vec{b} \mid\right.$ different $)=\frac{1}{\left(f_{\max }^{\prime}-f_{\min }^{\prime}\right)^{D}\left(b_{\max }-b_{\min }\right)^{D}}$.

As noted above, in principle the priors should be proper. However, for this problem the normalization factors in the densities $P(f, \vec{b} \mid$ same $)$ and $P\left(\vec{f}^{\prime}, \vec{b} \mid\right.$ different) cancel in Equation (A4) and we can finesse the issue of improper priors. But this is only because $f_{\max }=f_{\max }^{\prime} \rightarrow 1$ and $f_{\min }=f_{\min }^{\prime} \rightarrow 0$.

In general, one wants to be sure that in higher dimensional problems such as the one considered here, $\mathcal{R}$ behaves properly. For instance, suppose that the upper limit on $f$ was not 1 but $\rho$ and, similarly, the upper limit on $f_{i}^{\prime}$ was $\xi$. The priors on $\vec{b}$ would cancel, but we would be left with an overall constant $\rho / \xi^{D}$ that depends on the number of dimensions, $D$. There are ways to ensure that $\mathcal{R}$ behaves as would be expected. For instance, one

22 We take limits of the relevant variables following the advice in Jaynes \& Bretthorst (2005). One reason for doing so is to handle variables which in the limit are defined on $[0, \infty)$. A flat prior is improper in this limit; that is, it does not integrate to one. can use a method proposed by Berger \& Pericchi (2001), where the priors are such that

$$
\begin{aligned}
\iint d f d \vec{b} P(f, \vec{b} \mid \text { same }) & =\iint d \vec{f}^{\prime} d \vec{b} P \\
& \times\left(\vec{f}^{\prime}, \vec{b} \mid \text { different }\right)=1
\end{aligned}
$$

and therefore $\mathcal{R}$ is guaranteed to make sense. Another is simply to check that $\mathcal{R}$ exhibits the behavior that would be expected from a Bayes factor. For instance, one would expect that as the uncertainties decrease (i.e., $N_{i} \rightarrow \infty$ and $M_{i} \rightarrow \infty$ ), a larger fraction of pairs with different intrinsic spectra would have $\mathcal{R}<1$ and a larger fraction of pairs with similar intrinsic spectra would have $\mathcal{R}>1$. We will come back to this point later when we show that the $\mathcal{R}$ used to calculate the similarity of spectra indeed behaves properly as the flux uncertainties go to $0 .{ }^{23}$

Collecting the terms, the full Bayes factor for the Poisson case becomes

$$
\begin{aligned}
\mathcal{R} & =\frac{P(\mathrm{~A}, \mathrm{~B} \mid \text { different })}{P(\mathrm{~A}, \mathrm{~B} \mid \mathrm{same})} \\
& =\frac{\prod_{i=1}^{D} \int_{0}^{1} d f_{i}^{\prime} \int_{0}^{\infty} d b_{i} \frac{\left(f_{i}^{\prime} b_{i}\right)^{N_{i}} e^{-f_{i}^{\prime} b_{i}}}{N_{i} !} \frac{\left(\left(1-f_{i}^{\prime}\right) b_{i}\right)^{M_{i}} e^{-\left(1-f_{i}^{\prime} b_{i}\right.}}{M_{i} !}}{\int_{0}^{1} d f \prod_{i=1}^{D} \int_{0}^{\infty} d b_{i} \frac{\left(f b_{i}\right)^{N_{i}} e^{-f b_{i}}}{N_{i} !} \frac{\left((1-f) b_{i}\right)^{M_{i}} e^{-(1-f) b_{i}}}{M_{i} !}},
\end{aligned}
$$

which can be evaluated to be

$$
\begin{aligned}
\mathcal{R} & =\frac{\prod_{i=1}^{D} \frac{1}{N_{i}+M_{i}+1}}{\int_{0}^{1} d f \prod_{i=1}^{D}\left(\begin{array}{c}
N_{i}+M_{i} \\
M_{i}
\end{array}\right) f^{N_{i}}(1-f)^{M_{i}}}, \\
& =\frac{\prod_{i=1}^{D} B\left(N_{i}+1, M_{i}+1\right)}{B(N+1, M+1)},
\end{aligned}
$$

where $B(n, m)$ is the beta function $B(n, m) \equiv \Gamma(n) \Gamma(m) / \Gamma(n+$ $m), N \equiv \sum_{i=1}^{D} N_{i}$, and $M \equiv \sum_{i=1}^{D} M_{i}$.

\section{A.3. Gaussian Errors}

In many cases (including ours), the data are in units of flux, not photon counts, and the flux distribution for the $i$ th bin will follow a Gaussian distribution. In this case, the likelihood in Equation (A5) is re-expressed as

$$
\begin{aligned}
P\left(\mathrm{~A}, \mathrm{~B} \mid \vec{f}^{\prime}, \vec{b}\right)= & \prod_{i=1}^{D} \frac{1}{\sqrt{2 \pi} \sigma_{A i}} e^{-\frac{\left[f_{i}^{\prime} b_{i}-F_{A i}\right]^{2}}{2 \sigma_{A i}^{2}}} \\
& \times \frac{1}{\sqrt{2 \pi} \sigma_{B i}} e^{-\frac{\left[\left(1-f_{i}^{\prime}\right) b_{i}-F_{B i}\right]^{2}}{2 \sigma_{B i}^{2}}}
\end{aligned}
$$

and the likelihood in Equation (A6) as

$$
\begin{aligned}
P(\mathrm{~A}, \mathrm{~B} \mid f, \vec{b})= & \prod_{i=1}^{D} \frac{1}{\sqrt{2 \pi} \sigma_{A i}} e^{-\frac{\left[f b_{i}-F_{A}\right]^{2}}{2 \sigma_{A i}}} \\
& \times \frac{1}{\sqrt{2 \pi} \sigma_{B i}} e^{-\frac{\left[(1-f) b_{i}-F_{B i}\right]^{2}}{2 \sigma_{B i}^{2}}}
\end{aligned}
$$

${ }^{23}$ If $\mathcal{R}$ were not a Bayes factor, this would not affect the results presented in this work as $\mathcal{R}$ is only used as a (very good) discriminant between spectral pairs whose intrinsic spectra are different and those whose intrinsic spectra are the same (see Figure 10). 
where, for the $i$ th wavelength bin, $f b_{i}$ and $f_{i}^{\prime} b_{i}$ are the mean fluxes for source $\mathrm{A},(1-f) b_{i}$ and $\left(1-f_{i}^{\prime}\right) b_{i}$ are the mean fluxes expected from source $\mathrm{B}, F_{A i}$ and $F_{B i}$ are the measured fluxes, and $\sigma_{A i}$ and $\sigma_{B i}$ are the errors on the measured fluxes, all in the $i$ th bin. Plugging Equations (A20) and (A21) into Equation (A18)

$$
\mathcal{R}=\frac{\prod_{i=1}^{D} \int_{0}^{1} d f_{i}^{\prime} \int_{0}^{\infty} d b_{i} \frac{1}{2 \pi \sigma_{A i} \sigma_{B i}} e^{-\frac{\left[f_{i}^{\prime} b_{i}-F_{A i}\right]^{2}}{2 \sigma_{A i}^{2}}} e^{-\frac{\left[\left(1-f_{i}^{\prime}\right) b_{i}-F_{B i}\right]^{2}}{2 \sigma_{B i}^{2}}}}{\int_{0}^{1} d f \prod_{i=1}^{D} \int_{0}^{\infty} d b_{i} \frac{1}{2 \pi \sigma_{A i} \sigma_{B i}} e^{-\frac{\left[f b_{i}-F_{A i}\right]^{2}}{2 \sigma_{A}^{2}}} e^{-\frac{\left[(1-f) b_{i}-F_{B i}\right]^{2}}{2 \sigma_{B i}^{2}}}},
$$

where now $b_{i}$ can be integrated semianalytically (see Section A.7).

\section{A.4. Including Extinction}

We also wish to account for the possible presence of a screen of cold dust in front of our sources. To do this, we use the carbonaceous-silicate grain model of Weingartner \& Draine (2001), their model "A," with $R_{V}=5.5$ and the grain abundances per $\mathrm{H}$ increased by a factor of 1.42 , although any dust extinction law can in principle be used. The effect of dust extinction is accounted for by a simple exponential factor, $e^{-k(\lambda) x}$, where $k(\lambda)$ is the absorption cross section per mass of dust $\left(\mathrm{cm}^{2} \mathrm{~g}^{-1}\right)$ as a function of wavelength, and $x$ is the column density $\left(\mathrm{g} \mathrm{cm}^{-2}\right)$.

Figure 19 shows the distribution of $e^{-k(\lambda) x}$ for an arbitrarily chosen value for $x$. If $x_{A}$ and $x_{B}$ are the column densities for sources $\mathrm{A}$ and $\mathrm{B}$, respectively, they are parameterized by $x_{A}=f_{e} b_{e}$ and $x_{B}=\left(1-f_{e}\right) b_{e}$, where the parameters $f_{e}$ and $b_{e}$ are analogous to the parameters $f_{i}^{\prime}$ and $b_{i}$ for the flux distribution considered above. That is, $b_{e}$ is the sum of the dust columns in front of sources $\mathrm{A}$ and $\mathrm{B}$, and $f_{e}$ is the fraction of the dust column in front of source A. The Bayes factor, Equation (A4), is then modified to include these new parameters

$$
\begin{aligned}
\mathcal{R}= & \left\{\int_{0}^{1} d f_{e} \int_{0}^{\infty} d b_{e} \int_{0}^{1} d \vec{f}^{\prime} \int_{0}^{\infty} d \vec{b} P\left(\mathrm{~A}, \mathrm{~B} \mid f_{e}, b_{e}, \vec{f}^{\prime}, \vec{b}\right)\right. \\
& \left.\times P\left(f_{e}, b_{e}, \vec{f}^{\prime}, \vec{b} \mid \text { different }\right)\right\} /\left\{\int_{0}^{1} d f_{e} \int_{0}^{\infty} d b_{e} \int_{0}^{1} d f\right. \\
& \left.\times \int_{0}^{1} \int_{0}^{\infty} d \vec{b} P\left(\mathrm{~A}, \mathrm{~B} \mid f_{e}, b_{e}, f, \vec{b}\right) P\left(f_{e}, b_{e}, f, \vec{b} \mid \text { same }\right)\right\},
\end{aligned}
$$

and the likelihood of parameters $f_{e}, b_{e}, \vec{f}^{\prime}$, and $\vec{b}$ is now

$$
\begin{aligned}
P\left(\mathrm{~A}, \mathrm{~B} \mid f_{e}, b_{e}, \vec{f}^{\prime}, \vec{b}\right)= & \prod_{i=1}^{D} \frac{1}{\sqrt{2 \pi} \sigma_{A i}} e^{-\frac{\left[e^{-k_{i} f_{e} b_{e}} f_{i}^{\prime} b_{i}-F_{A i}\right]^{2}}{2 \sigma_{A i}^{2}}} \\
& \times \frac{1}{\sqrt{2 \pi} \sigma_{B i}} e^{-\frac{\left[-e^{-k_{i}\left(1-f_{e}\right) b_{e}}\left(1-f_{i}^{\prime}\right) b_{i}-F_{B i}\right]^{2}}{2 \sigma_{B i}^{2}}}
\end{aligned}
$$

while the likelihood of parameters $f_{e}, b_{e}, f$, and $\vec{b}$ is

$$
\begin{aligned}
P\left(\mathrm{~A}, \mathrm{~B} \mid f_{e}, b_{e}, f, \vec{b}\right)= & \prod_{i=1}^{D} \frac{1}{\sqrt{2 \pi} \sigma_{A i}} e^{-\frac{\left[e^{-k_{i} f_{e} b_{e}} f b_{i}-F_{A i}\right]^{2}}{2 \sigma_{A i}^{2}}} \\
& \times \frac{1}{\sqrt{2 \pi} \sigma_{B} i} e^{-\frac{\left[-e^{-k_{i}\left(1-f_{e}\right) b_{e}}(1-f) b_{i}-F_{B i}\right]^{2}}{2 \sigma_{B i}^{2}}},
\end{aligned}
$$

where $k_{i}$ is the absorption coefficient in the $i$ th wavelength bin. In other words, the mean flux obtained from source A with no dust is $f_{i}^{\prime} b_{i}$ and $f b_{i}$ for the "different" and "same" hypotheses, respectively. If dust is included, ${ }^{24}$ this flux would be attenuated by an additional factor, $e^{-k_{i} f_{e} b_{e}}$.

In practice, to calculate $\mathcal{R}$, Equation (A23) needs to be constrained as it has too many degrees of freedom. Let us assume that some maximum, $b_{e \max }$, has been chosen so that $b_{e}<b_{e \max }$. The priors are then modified

$$
P\left(f_{e}, b_{e}, f, \vec{b} \mid \text { same }\right) \rightarrow \frac{\Theta\left(b_{e \max }-b_{e}\right)}{b_{e \max }\left(f_{\max }-f_{\min }\right)\left(b_{\max }-b_{\min }\right)^{D}}
$$

and

$$
\begin{aligned}
& P\left(f_{e}, b_{e}, \vec{f}^{\prime}, \vec{b} \mid \text { different }\right) \\
& \quad \rightarrow\left\{\Theta\left(b_{e \max }-b_{e}\right)\right\} /\left\{b_{e \max }\left(f_{\max }^{\prime}-f_{\min }^{\prime}\right)^{D}\left(b_{\max }-b_{\min }\right)^{D}\right\},
\end{aligned}
$$

where $\Theta$ is defined as

$$
\Theta\left(b_{e \max }-b_{e}\right)= \begin{cases}1, & b_{e \max } \geqslant b_{e} \\ 0, & b_{e \max }<b_{e} .\end{cases}
$$

Putting everything together, the full likelihood then becomes

$$
\begin{aligned}
\mathcal{R}= & \left\{\int_{0}^{1} d f_{e} \int_{0}^{\infty} d b_{e} \int_{0}^{1} d \vec{f}^{\prime} \int_{0}^{\infty} d \vec{b} P\left(\mathrm{~A}, \mathrm{~B} \mid f_{e}, b_{e}, \vec{f}^{\prime}, \vec{b}\right)\right. \\
& \left.\times P\left(f_{e}, b_{e}, \vec{f}^{\prime}, \vec{b} \mid \text { different }\right)\right\} /\left\{\int_{0}^{1} d f_{e} \int_{0}^{\infty} d b_{e} \int_{0}^{1} d f\right. \\
& \left.\times \int_{0}^{1} \int_{0}^{\infty} d \vec{b} P\left(\mathrm{~A}, \mathrm{~B} \mid f_{e}, b_{e}, f, \vec{b}\right) P\left(f_{e}, b_{e}, f, \vec{b} \mid \text { same }\right)\right\} \\
= & \left\{\int _ { 0 } ^ { 1 } d f _ { e } \int _ { 0 } ^ { b _ { e \operatorname { m a x } } } d b _ { e } \prod _ { i = 1 } ^ { D } \int _ { 0 } ^ { 1 } d f _ { i } ^ { \prime } \int _ { 0 } ^ { \infty } d b _ { i } \operatorname { e x p } \left(-\left\{\left[e^{-k_{i} f_{e} b_{e}}\right.\right.\right.\right. \\
& \left.\left.\left.\left.\times f_{i}^{\prime} b_{i}-F_{A i}\right]^{2}\right\} /\left\{2 \sigma_{A i}^{2}\right\}\right)\right\} /\left\{\int_{0}^{1} d f_{e} \int_{0}^{b_{e m a x}} d b_{e}\right. \\
& \times \int_{0}^{1} d f \prod_{i=1}^{D} \int_{0}^{\infty} d b_{i} \exp \left(-\left\{\left[e^{-k_{i} f_{e} b_{e}} f b_{i}-F_{A i}\right]^{2}\right\} /\right. \\
& \left.\left.\left\{2 \sigma_{A i}^{2}\right\}\right)\right\} \times \frac{\exp \left(-\frac{\left[e^{-k_{i}\left(1-f_{e}\right) b_{e}}\left(1-f_{i}^{\prime}\right) b_{i}-F_{B i}\right]^{2}}{2 \sigma_{B i}^{2}}\right)}{\exp \left(-\frac{\left[e^{-k_{i}\left(1-f_{e}\right) b_{e}}(1-f) b_{i}-F_{B i}\right]^{2}}{2}\right)} .
\end{aligned}
$$

Parameter $b_{i}$ can be integrated semianalytically as shown in Section A.7. Both the numerator and the denominator of Equation (A29) are calculated using adaptive Monte Carlo integration methods, VEGAS and miser (Press et al. 1992).

There is a degeneracy in Equation (A29). Suppose we are comparing two spectra which both have the shape of the extinction curve shown in Figure 19. In this case, the best estimate of the parameters can come from an "intrinsic" $\operatorname{spectrum}(\sim \vec{b})$ that looks like the extinction curve and is not propagated through any dust, or that is flat and is propagated through dust (assuming that dust follows the extinction curve in Figure 19). In practice,

\footnotetext{
${ }^{24}$ We have parameterized the dust in such a way that one could in principle ask the question whether or not two sources have the same column density. Under the "same" hypothesis $f_{e}=1 / 2$, while under the "different" hypothesis
} $f_{e}$ would be marginalized. However, we do not consider this question here. 


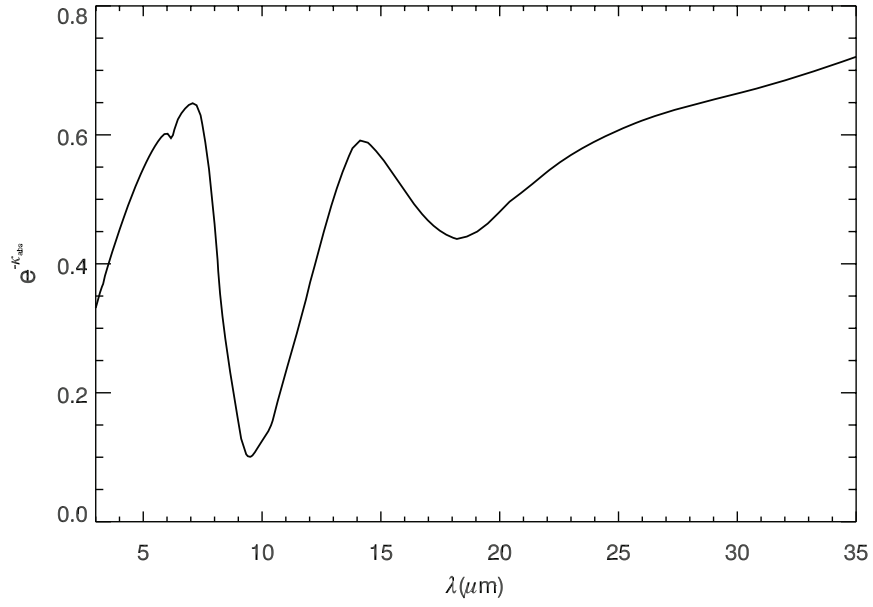

Figure 19. The dust attenuation factor, $e^{-k_{\mathrm{abs}}(\lambda) x}$, for an arbitrarily chosen value of $x$.

this situation is exceedingly unlikely, as the intrinsic spectra (which enter through $\vec{b}$ ), the relative normalizations (that enter through $f$ ) and the extinction correction all do the best they can to make a good fit. There are, however, examples where effects of the source distribution taking on characteristics of the dust to make the best fit can be seen; see, e.g., the comparison between IRAS 12071-0444 and IRAS 17179+5444, where the extinction is close to zero, but the spectra follow the features of the extinction curve in Figure 19.

Ideally, we would like a model for the intrinsic dust distribution in each ULIRG such that all the dust, hot and cold, can be placed where it should be (in the extinction parameters $f_{e}$ and $b_{e}$ ) and the source where it should be (in $f$ and $\vec{b}$ ). Such a model does not, however, exist, and so the best we can do is attempt to account for differences solely due to cold foreground dust. While this is undoubtedly an improvement on not attempting to account for dust at all, it does mean that (without constraints on the shape of the source distribution) the contributions of dust and the source may not be proportioned correctly.

\section{A.5. Behavior of $\mathcal{R}$}

As discussed previously, $\mathcal{R}$ must behave as a Bayes factor should-i.e., as $\sigma_{A i} \rightarrow 0$ and $\sigma_{B i} \rightarrow 0$, pairs with like intrinsic spectra should all have $\mathcal{R}$ 's less than 1 and pairs with unlike intrinsic spectra should have $\mathcal{R}$ 's larger than 1 . To this end, Figure 10 shows that the spectra with lower errors systematically have a larger separation in $\mathcal{R}$ than those with larger errors, indicating the likelihood is behaving as expected, although this separation is somewhat obscured by the decreasing accuracy/ precision of the Monte Carlo integration as the uncertainties decrease.

To gather further intuition about the behavior of $\mathcal{R}$, we study the case where the Gaussian (statistical) fluctuations in the data are smaller than what is assumed in the calculation. We generate pairs of spectra with the same intrinsic spectra but different dust extinctions. The two spectra are then fluctuated independently assuming $2 \%$ Gaussian uncertainties; $\mathcal{R}$ is then calculated for each pair assuming $5 \%$ uncertainties. As there would be less variation in the data than expected by the calculation, one would expect the spectra to look more similar than if the fluctuations in the data were, say, $\sim 5 \%$. Therefore, one would expect $\mathcal{R}$ to be less than if the fluctuations in the data were indeed $\sim 5 \%$. Figure 20 shows that $\mathcal{R}$ behaves as we expect where those data

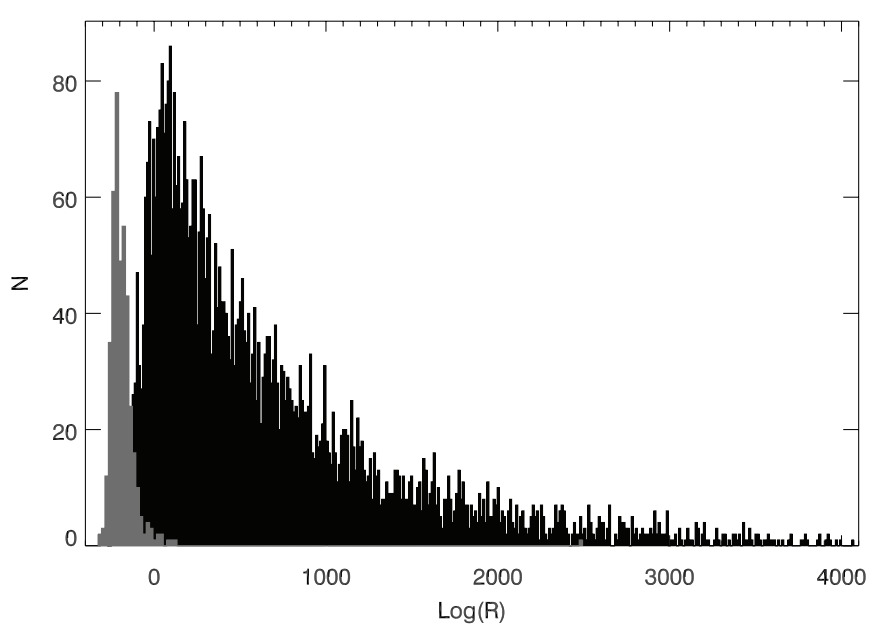

Figure 20. Distributions of $\mathcal{R}$ calculated on spectral pairs with the same intrinsic spectrum but different dust extinctions. The black histogram is for those randomly generated spectral pairs with $5 \%$ Gaussian uncertainties while the gray histogram is for those pairs with $2 \%$ uncertainties. As expected, those pairs with "true" uncertainties less than those used in the calculation of $\mathcal{R}$ are systematically shifted to smaller values.

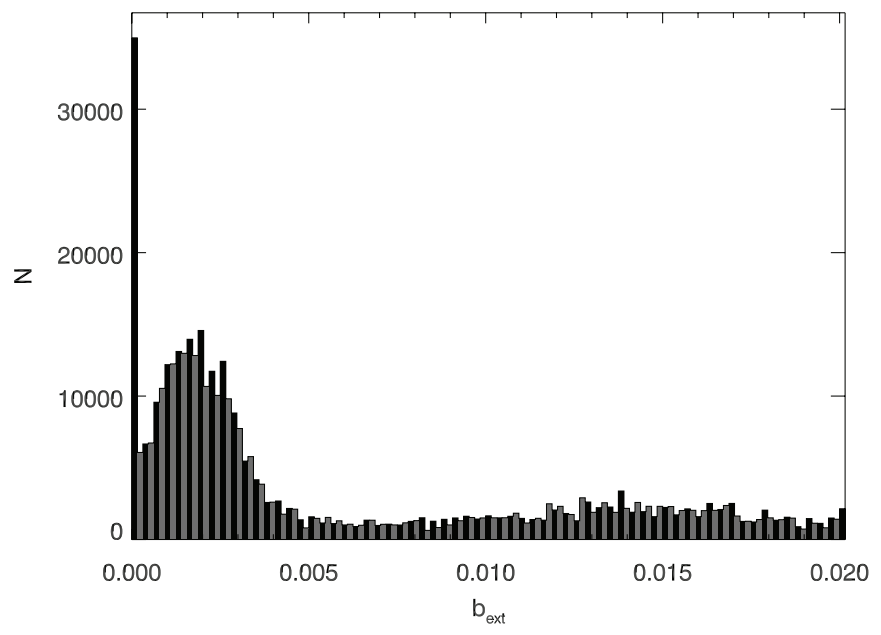

Figure 21. The distribution of values of $b_{e}$ resulting from maximizing Equation (A30) for all possible pairs of spectra. $b_{e}$ is defined as the best estimate of the sum of the column densities of the two ULIRGs that obtains the best-fitting intrinsic spectra.

with $2 \%$ uncertainties are systematically shifted to lower values than those with $5 \%$ uncertainties.

\section{A.6. Maximum A Posteriori (MAP) Probability}

We would like to maximize the a posteriori probability defined as the unmarginalized denominator in Equation (A29) (which accounts for the probability that the two spectra come from the sources with the same intrinsic spectrum). This is done for several reasons. First, maximizing this likelihood is one way of finding out how similar the two spectra are. Second, by maximizing a posteriori probability with respect to $f_{e}, b_{e}, f$, and $\vec{b}$, one can obtain a best estimate of the mean distributions of these parameters for spectra A and B. Third, by plotting the best estimate of $b_{e}$ for all pairing of the spectra, we get an estimate for the upper limit for $b_{e}$.

The third point is worth describing in more detail. The rationale for calculating the upper limit on $b_{e}$ in this way is as follows. We start by (falsely) supposing that the intrinsic sources are the same, and that the only thing that differentiates 
them is an overall flux normalization and the amount of dust in front of them. We then calculate the best estimate for $b_{e}$ by finding the maximum a posteriori (MAP) probability defined later in the text (Equation (A30)), thereby creating a distribution of the best estimates for $b_{e}$ for our data sample. The maximum $b_{e}$ found from this distribution is an estimate of the maximum $b_{e}$ that might be found for any of the pairs of objects. If our assumption that the sources were the same was incorrect, the differences in the sources would randomize the $b_{e}$ distribution to some degree, thus effectively only pushing the upper limit on $b_{e}$ higher. That is, the "true" value of $b_{e}$ is likely to be covered in the integration of $b_{e}$.

One could pose two objections to this method: (1) for a given pair of objects, we are using it in the distribution of the best estimates for $b_{e}$ to find the upper limit of $b_{e}$ and then subsequently using that upper limit to calculate $\mathcal{R}$ with the very same pair, and (2) we are not using the shape of the distribution of $b_{e}$ as a prior when calculating $\mathcal{R}$. The first objection can be addressed by realizing that in our sample there are 5151 pairings, and the effect of using our knowledge of $b_{e}$ from a given pair twice (once to develop the distribution of the best estimates for $b_{e}$, and once for calculating the result using our knowledge on the upper limit of $b_{e}$ ) is small. As for the latter objection, one can argue that the distribution is skewed by the fact that the intrinsic source spectra for the pairs are different and therefore a flat prior on $b_{e}$ is at least as good an estimate of our a priori knowledge of $b_{e}$ as a prior that takes into account the shape of the $b_{e}$ distribution.

We now turn back to the a posteriori probability defined as

$$
\begin{aligned}
L\left(\mathrm{~A}, \mathrm{~B} \mid f_{e}, b_{e}, f, \vec{b}\right) \equiv & P\left(\mathrm{~A}, \mathrm{~B} \mid f_{e}, b_{e}, f, \vec{b}\right) \\
& \times P\left(f_{e}, b_{e}, f, \vec{b} \mid \text { same }\right) .
\end{aligned}
$$

Maximizing Equation (A30) is equivalent to minimizing

$$
\begin{aligned}
- & \log \left(L\left(\mathrm{~A}, \mathrm{~B} \mid f_{e}, b_{e}, f, \vec{b}\right)\right) \\
\equiv & -\log \left[P\left(\mathrm{~A}, \mathrm{~B} \mid f_{e}, b_{e}, f, \vec{b}\right) P\left(f_{e}, b_{e}, f, \vec{b} \mid \text { same }\right)\right] \\
= & \sum_{i=1}^{D} \frac{\left[e^{-k_{i} f_{e} b_{e}} f b_{i}-F_{A i}\right]^{2}}{2 \sigma_{A i}^{2}} \\
& +\frac{\left[e^{-k_{i}\left(1-f_{e}\right) b_{e}}(1-f) b_{i}-F_{B i}\right]^{2}}{2 \sigma_{B i}^{2}}+\log \left(2 \pi \sigma_{A i} \sigma_{B i}\right) .
\end{aligned}
$$

Maximizing Equation (A30) (or minimizing Equation (A31)) yields a distribution of $b_{e}$ from all the pairs of objects considered in this work, from where one can empirically determine the limit on $b_{e}, b_{e}$ max. The distribution is shown in Figure 21. We chose $b_{e \max }=0.02$ as the nominal value as $b_{e}<b_{e \max }$ contains the bulk of spectral pairs, and $b_{e \max }=0.03$ to test the dependence of the network diagrams on dust extinction (see Figure 14).

The column densities found by minimizing Equation (A31) should not be regarded as good estimates of the "true" column densities because there is degeneracy in the intrinsic shapes of the spectra and the effects of extinction. For instance, two spectra that are intrinsically the same will yield the same measured spectra (within statistical uncertainties) provided there is no dust extinction. However, two ULIRGs may have different intrinsic spectra and suffer different amounts of dust extinctions, but the combination might conspire to make the measured spectra look similar. Also, as discussed above, ULIRGs with similar column densities might, in fact, render a low value for $b_{e}$ while the intrinsic spectra take on characteristics of the dust as there are no constraints imposed on them.

\section{A.7. Integrations with Respect to $\vec{b}$}

Integrating the Gaussians in Equation (A29) with respect to $b_{i}$ (Gradshteyn \& Rhyzik 2000), we obtain

$$
\begin{aligned}
& \mathcal{R}=\frac{\int_{0}^{1} d f_{e} \int_{0}^{b_{e} \max } d b_{e} \prod_{i=1}^{D} \int_{0}^{1} d f_{i}^{\prime} \sqrt{\frac{\pi}{\left.2 \frac{f_{i}^{\prime 2} e^{-2 k_{i} f_{e} b_{e}}}{\sigma_{A i}^{2}}+\frac{\left(1-f_{i}^{\prime}\right)^{2} e^{-2 k_{i}\left(1-f_{e}\right) b_{e}}}{\sigma_{B i}^{2}}\right]}}}{\int_{0}^{1} d f_{e} \int_{0}^{b_{e} \max } d b_{e} \int_{0}^{1} d f \prod_{i=1}^{D} \sqrt{\left.\frac{\pi}{2\left[\frac{f^{2} e^{-2 k_{i} f_{e} b_{e}}}{\sigma_{A i}^{2}}+\frac{(1-f)^{2} e^{2}-2 k_{i}\left(1-f_{e} b_{e}\right.}{\sigma_{B i}^{2}}\right]}\right]}}
\end{aligned}
$$

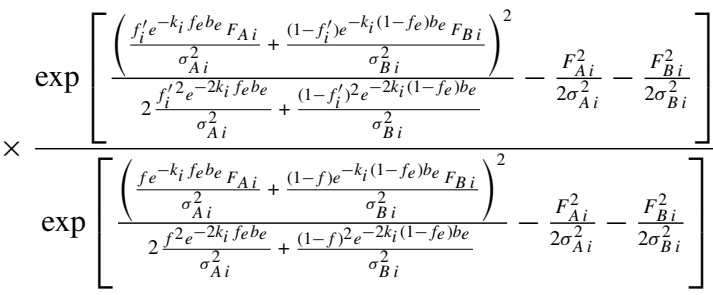

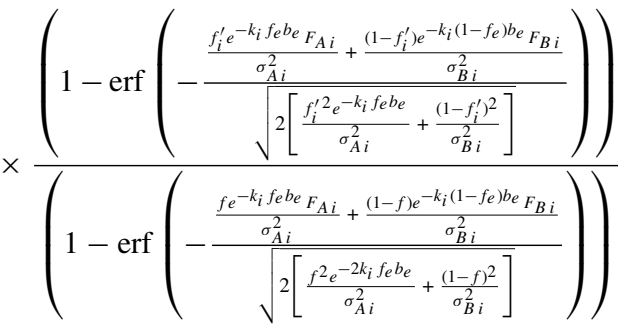

where $\operatorname{erf}(u)$ is defined as

$$
\operatorname{erf}(u) \equiv \frac{2}{\sqrt{\pi}} \int_{0}^{u} e^{-x^{2}} d x
$$

\section{REFERENCES}

Allen, D. A., Norris, R. P., Meadows, V. S., \& Roche, P. F. 1991, MNRAS, 248, 528

Armus, L., Heckman, T. M., \& Miley, G. K. 1989, ApJ, 347, 727

Armus, L., et al. 2004, ApJS, 154, 178

Armus, L., et al. 2006, ApJ, 640, 204

Barger, A. J., Cowie, L. L., Sanders, D. B., Fulton, E., Taniguchi, Y., Sato, Y., Kawara, K., \& Okuda, H. 1998, Nature, 394, 248

Berger, J., \& Pericchi, L. 2001, in Model Selection (Monograph Series, Vol. 38), ed. P. Lahiri (Beachwood, OH: Institute of Mathematical Statistics)

Barnes, J. E., \& Hernquist, L. 1992, ARA\&A, 30, 705

Bergvall, N., Johansson, L., \& Olofsson, K. 1986, A\&A, 166, 92

Berta, S., et al. 2007, A\&A, 467, 565

Bianchi, S., Chiaberge, M., Piconcelli, E., Guainazzi, M., \& Matt, G. 2008, MNRAS, 386, 105

Blain, A. W., Chapman, S. C., Smail, I., \& Ivison, R. 2004, ApJ, 611, 725

Borys, C., Chapman, S., Halpern, M., \& Scott, D. 2003, MNRAS, 344, 385

Borys, C., et al. 2006, ApJ, 636, 134

Braito, V., Reeves, J. N., Della Ceca, R., Ptak, A., Risaliti, G., \& Yaqoob, T. 2009, A\&A, accepted (arXiv:0905.1041)

Brandes, U. 2001, J. Math. Sociol., 25, 163

Bridge, C. R., et al. 2007, ApJ, 659, 931

Bushouse, H. A., et al. 2002, ApJS, 138, 1

Chapman, S. C., Windhorst, R., Odewahn, S., Yan, H., \& Conselice, C. 2003, ApJ, 599, 92

Connolly, B. M., et al. 2006, Phys. Rev. D, 74, 043001

Cui, J., Xia, X.-Y., Deng, Z.-G., Mao, S., \& Zou, Z.-L. 2001, AJ, 122, 63

Darling, J., \& Giovanelli, R. 2006, AJ, 132, 2596

Dasyra, K. M., et al. 2006a, ApJ, 638, 745

Dasyra, K. M., et al. 2006b, ApJ, 651, 835

Desai, V., et al. 2007, ApJ, 669, 810

Dole, H., et al. 2001, A\&A, 372, 364

Dubinski, J., Mihos, J. C., \& Hernquist, L. 1999, ApJ, 526, 607

Duc, P.-A., Mirabel, I. F., \& Maza, J. 1997, A\&AS, 124, 533 
Erdos, P., \& Renyi, A. 1959, Publ. Math. Debrecen, 6, 290

Farrah, D., Afonso, J., Efstathiou, A., Rowan-Robinson, M., Fox, M., \& Clements, D. 2003, MNRAS, 343, 585

Farrah, D., Verma, A., Oliver, S., Rowan-Robinson, M., \& McMahon, R. 2002, MNRAS, 329, 605

Farrah, D., et al. 2001, MNRAS, 326, 1333

Farrah, D., et al. 2006, ApJ, 641, L17

Farrah, D., et al. 2007, ApJ, 667, 149

Farrah, D., et al. 2008, ApJ, 677, 957

Franceschini, A., et al. 2003, MNRAS, 343, 1181

Freeman, L. C. 1979, Social Netw., 1, 215

Fruchterman, T. M. J., \& Reingold, E. M. 1991, Softw.: Pract. Exp., 21, 1129

Genzel, R., Tacconi, L. J., Rigopoulou, D., Lutz, D., \& Tecza, M. 2001, ApJ, 563,527

Genzel, R., et al. 1998, ApJ, 498, 579

Gradshteyn, I. S., \& Rhyzik, I. M. 2000, in Table of Integrals, Series and Products, ed. A. Jeffrey \& D. Zwillinger (6th ed.; New York: Academic Press)

Hao, L., et al. 2005, ApJ, 625, L75

Higdon, S. J. U., Armus, L., Higdon, J. L., Soifer, B. T., \& Spoon, H. W. W. 2006, ApJ, 648, 323

Houck, J. R., et al. 2004, ApJS, 154, 18

Huang, J. S., et al. 2009, ApJ, accepted (arXiv:0904.4479)

Hughes, D. H., et al. 1998, Nature, 394, 241

Imanishi, M., Dudley, C. C., Maiolino, R., Maloney, P. R., Nakagawa, T., \& Risaliti, G. 2007, ApJS, 171, 72

Jaynes, E. T., \& Bretthorst, G. L. (ed.) 2005, in Probability Theory: The Logic of Science (Cambridge: Cambridge Univ. Press)

Jeffreys, H. 1961, Theory of Probability (3rd edn.; Oxford: Oxford Univ. Press)

Kamada, T., \& Kawai, S. 1989, Inf. Proc. Lett., 31, 7

Kawakatu, N., Anabuki, N., Nagao, T., Umemura, M., \& Nakagawa, T. 2006, ApJ, 637, 104

Kawakatu, N., Imanishi, M., \& Nagao, T. 2007, ApJ, 661, 660

Kewley, L. J., Heisler, C. A., Dopita, M. A., \& Lumsden, S. 2001, ApJS, 132, 37

Kim, D.-C., \& Sanders, D. B. 1998, ApJS, 119, 41

Klaas, U., et al. 2001, A\&A, 379, 823

Lawrence, A., et al. 1999, MNRAS, 308, 897

Leech, K. J., Rowan-Robinson, M., Lawrence, A., \& Hughes, J. D. 1994, MNRAS, 267, 253

Lípari, S., Terlevich, R., Zheng, W., Garcia-Lorenzo, B., Sanchez, S. F., \& Bergmann, M. 2005, MNRAS, 360, 416

Lonsdale, C. J., Farrah, D., \& Smith, H. E. 2006, in Astrophysics Update 2, ed. John W. Mason (Heidelberg: Springer), 285

Lonsdale, C. J., et al. 2009, ApJ, 692, 422

Lusseau, D., \& Newman, M. E. J. 2004, Proc. R. So. B, 271, S477

Magliocchetti, M., Silva, L., Lapi, A., de Zotti, G., Granato, G. L., Fadda, D., \& Danese, L. 2007, MNRAS, 375, 1121

Melbourne, J., et al. 2008, AJ, 135, 1207

Meusinger, H., Stecklum, B., Theis, C., \& Brunzendorf, J. 2001, A\&A, 379 845

Mirabel, I. F., Lutz, D., \& Maza, J. 1991, A\&A, 243, 367
Mortier, A. M. J., et al. 2005, MNRAS, 363, 563

Pastor-Satorras, R., Vazquez, A., \& Vespignani, A. 2001, Phys. Rev. Lett., 87, 258701

Press, W. H., Teukolsky, S. A., Vetterling, W. T., \& Flannery, B. P. 1992, Numerical Recipes in C (Cambridge: Cambridge Univ. Press)

Rigopoulou, D., Spoon, H. W. W., Genzel, R., Lutz, D., Moorwood, A. F. M., \& Tran, Q. D. 1999, AJ, 118, 2625

Rowan-Robinson, M., et al. 1997, MNRAS, 289, 490

Rupke, D. S., Veilleux, S., \& Sanders, D. B. 2005, ApJS, 160, 87

Sanders, D. B., \& Mirabel, I. F. 1996, ARA\&A, 34, 749

Sekiguchi, K., \& Wolstencroft, R. D. 1993, MNRAS, 263, 349

Sellke, T., Bayarri, M. J., \& Berger, J. O. 2001, Am. Stat., 55, 62

Siganos, G., Faloutsos, M., Faloutsos, P., \& Faloutsos, C. 2003, ACM/IEEE Trans. Netw., 11, 514

Sirocky, M. M., Levenson, N. A., Elitzur, M., Spoon, H. W. W., \& Armus, L. 2008, ApJ, 678, 729

Sivia, D. S. 1996, Data Analysis: A Bayesian Tutorial (Oxford: Oxford Univ. Press)

Smail, I., Chapman, S. C., Blain, A. W., \& Ivison, R. J. 2004, ApJ, 616, 71

Soifer, B. T., Helou, G., \& Werner, M. 2008, ARA\&A, 46, 201

Soifer, B. T., et al. 1984, ApJ, 278, L71

Spoon, H. W. W., Armus, L., Marshall, J. A., Bernard-Salas, J., Farrah, D. Charmandaris, V., \& Kent, B. R. 2009, ApJ, 693, 1223

Spoon, H. W. W., Marshall, J. A., Houck, J. R., Elitzur, M., Hao, L., Armus, L., Brandl, B. R., \& Charmandaris, V. 2007, ApJ, 654, L49

Spoon, H. W. W., et al. 2004, ApJS, 154, 184

Spoon, H. W. W., et al. 2006, ApJ, 638, 759

Stanford, S. A., Stern, D., van Breugel, W., \& De Breuck, C. 2000, ApJS, 131, 185

Strauss, M. A., Davis, M., Yahil, A., \& Huchra, J. P. 1990, ApJ, 361, 49

Surace, J. A., Sanders, D. B., \& Evans, A. S. 2000, ApJ, 529, 170

Tacconi, L. J., Genzel, R., Lutz, D., Rigopoulou, D., Baker, A. J., Iserlohe, C., \& Tecza, M. 2002, ApJ, 580, 73

Takata, T., Sekiguchi, K., Smail, I., Chapman, S. C., Geach, J. E., Swinbank, A. M., Blain, A., \& Ivison, R. J. 2006, ApJ, 651, 713

Taniguchi, Y., Ikeuchi, S., \& Shioya, Y. 1999, ApJ, 514, L9

Tran, Q. D., et al. 2001, ApJ, 552, 527

Tremaine, S., et al. 2002, ApJ, 574, 740

Valiante, E., Lutz, D., Sturm, E., Genzel, R., Tacconi, L. J., Lehnert, M. D., \& Baker, A. J. 2007, ApJ, 660, 1060

Vega, O., Clemens, M. S., Bressan, A., Granato, G. L., Silva, L., \& Panuzzo, P. 2008, A\&A, 484, 631

Veilleux, S., Kim, D.-C., \& Sanders, D. B. 1999, ApJ, 522, 113

Veilleux, S., Kim, D.-C., \& Sanders, D. B. 2002, ApJS, 143, 315

Veilleux, S., Kim, D.-C., Sanders, D. B., Mazzarella, J. M., \& Soifer, B. T. 1995, ApJS, 98, 171

Veilleux, S., Sanders, D. B., \& Kim, D.-C. 1999, ApJ, 522, 139

Veilleux, S., et al. 2006, ApJ, 643, 707

Véron-Cetty, M.-P., \& Véron, P. 2001, A\&A, 374, 92

Weingartner, J. C., \& Draine, B. T. 2001, ApJ, 548, 296

Werner, M. W., et al. 2004, ApJS, 154, 1 